\title{
Intraoperative Monitoring of the Obese Patient Undergoing Surgery: A Narrative Review
}

\author{
Andrea P. Haren · Shrijit Nair · Maria C. Pace - Pasquale Sansone
}

Received: February 17, 2021 / Accepted: May 5, 2021

(C) The Author(s) 2021

\begin{abstract}
With the increasing prevalence of obesity in the population, anaesthetists must confidently manage both the pathophysiological and technical challenges presented in bariatric and nonbariatric surgery. The intraoperative period represents an important opportunity to optimise and mitigate risk. However, there is little formal guidance on what intraoperative monitoring techniques should be used in this population. This narrative review collates the existing evidence for intraoperative monitoring devices in the obese patients. Although a number of non-invasive blood pressure monitors have been tested, an invasive arterial line remains the most reliable monitor if accurate, continuous monitoring is required. Goal-directed fluid therapy is recommended by clinical practice guidelines, but the methods tested to assess this had guarded applicability to the obese population. Transcutaneous carbon dioxide $\left(\mathrm{CO}_{2}\right)$ monitoring may offer additional benefit to standard capnography in this population. Individually titrated positive end
\end{abstract}

A. P. Haren · S. Nair

Department of Anaesthesia, St Vincent's University

Hospital, Dublin 4, Dublin, Ireland

M. C. Pace $\cdot$ P. Sansone $(\square)$

University of Campania "Luigi Vanvitelli", Naples,

Italy

e-mail: pasquale.sansone@unicampania.it expiratory pressure (PEEP) and recruitment manoeuvres improved intraoperative mechanics but yielded no benefit in the immediate postoperative period. Depth of anaesthesia monitoring appears to be beneficial in the perioperative period regarding recovery times and complications. Objective confirmation of reversal of neuromuscular blockade continues to be a central tenet of anaesthesia practice, particularly relevant to this group who have been characterised as an "at risk" extubation group. Where deep neuromuscular blockade is used, continuous neuromuscular blockade is suggested. Both obesity and the intraoperative context represent somewhat unstable search terms, as the clinical implications of the obesity phenotype are not uniform, and the type and urgency of surgery have significant impact on the intraoperative setting. This renders the generation of summary conclusions around what intraoperative monitoring techniques are suitable in this population highly challenging.

Keywords: Intraoperative; Monitoring; Obese; Obesity; Perioperative 


\section{Key Summary Points}

With the increasing prevalence of obesity, anaesthetists must be able to confidently manage the associated pathophysiological and technical challenges.

There has been an emphasis on preoperative optimisation and postoperative disposition for this potentially high-risk population.

However, little to date has been published specific to the intraoperative monitoring of this cohort. We summarise the published literature on this topic.

While a range of intraoperative monitoring techniques have been tested, there is guarded applicability of more novel tools (e.g. continuous non-invasive blood pressure monitors) to this population. We currently cannot recommend any changes to standard monitoring practices.

\section{DIGITAL FEATURES}

This article is published with digital features, including a summary slide, to facilitate understanding of the article. To view digital features for this article go to https://doi.org/10.6084/ m9.figshare.14535288.

\section{INTRODUCTION}

Worldwide, the prevalence of obesity has nearly tripled in the past 40 years [1]. Comorbidities are more frequent in the obese population, including those requiring surgical intervention. It is estimated that approximately $30 \%$ of the general surgical population presents with obesity [2]. With an improved safety profile, and evidence to support its long-term efficacy, the volume of bariatric surgery is expected to increase [3]. Meanwhile, the number of obese

patients presenting for non-bariatric surgery continues to grow, demanding expertise of all anaesthetists. Obesity has profound pathophysiological consequences as well as technical challenges of which the anaesthetist must be cognisant. A number of guidelines have been issued which address the perioperative management of the obese patient undergoing surgery [4-7]. A key aspect of these is the drive to facilitate the inclusion of obese patients in ambulatory lists, and enhanced recovery protocols, all of which tend towards minimally invasive strategies for monitoring. These consensus statements have contributed greatly towards the safe management of the patient, with particular focus on the preoperative identification and optimisation of co-morbidities, appropriate equipment in the operating theatre, and a dedicated plan for postoperative disposition. However, while there are published guidelines on the minimum monitoring standards for anaesthesia and recovery [8], there has been little published to date specific to the intraoperative monitoring of the obese patient. This is despite the fact that optimal intraoperative management represents an opportunity for mitigation and avoidance of complications postoperatively. This has been practically realised in terms of, for example, opioid-sparing techniques. The objective of this narrative review is to present and summarise the evidence regarding the intraoperative monitoring of the obese patient.

\section{METHODS}

\section{Ethics}

Our study did not require ethical board approval as it did not contain human or animal trials.

\section{Identification of Studies/Data Sources}

The MEDLINE electronic database via the Ovid Interface was searched using a prespecified search strategy. This was formulated according to the Population, Intervention, Comparison, 
Outcome (PICO) criteria, with target population constituting obese patients undergoing surgery and intervention being description of intraoperative monitoring technique $[\mathrm{MeSH}$ search terms: obese OR obesity AND intraoperative monitoring]. Additional eligible studies were retrieved by hand searching bibliographies of relevant articles. The search was limited to English language articles only, with date restriction to exclude studies published prior to 2010. The last electronic search was performed on 29 December 2020.

\section{Study Selection}

Article abstracts and subsequently the full text articles were independently assessed for eligibility by two researchers (AH and $\mathrm{SN}$ ), with any discrepancies referred to corresponding author (PS) for resolution.

The criteria for inclusion were: (1) randomised controlled trials, quasi-experimental and prospective or retrospective observational studies; (2) adults (over 18 years); (3) obese patients; (4) examination of intraoperative monitoring technique.

Exclusion criteria were; (1) case reports, case studies, editorials, review articles, and conference abstracts; (2) obstetrics.

\section{Data Extraction}

Two reviewers ( $\mathrm{AH}$ and $\mathrm{SN}$ ) extracted data from eligible full-text articles to collate information on study characteristics and outcome measures (Fig. 1).

\section{Data Synthesis}

Anticipated heterogeneity between studies precluded meta-analysis; therefore, the a priori decision to conduct narrative synthesis was taken.

All identified papers were first read and reread several times with the key points recorded to ensure familiarity with the literature. All included studies were tabulated and translated using content analysis. Relationships in the data were explored using grouping and textual descriptions. The robustness of the synthesis was evaluated and presented using critical reflection on the synthesis process.

\section{RESULTS}

The majority of studies retrieved concerned the cardiovascular $(n=14)$ [9-22], respiratory $(n=7)$ [23-29], and neurological $(n=9)$ [30-37] systems. Two pertained to point-of-care testing $[38,39]$. These were tabulated according to theme (Tables 1, 2, 3, 4, 5, 6, 7, 8, 9, hereafter). The remainder of the studies belonged to miscellaneous systems (renal $n=1[40]$, endocrine $n=10[41])$ and are alluded to individually in the discussion. One group reported the results of their enhanced recovery after a bariatric surgery protocol, including specific recommendations around intraoperative monitoring [42].

\section{DISCUSSION}

A systems-based approach to reviewing the results was applied, in accordance with the themes which emerged on tabulating the studies.

\section{Cardiovascular}

At a population level, obesity is an established risk factor for cardiovascular disease [43]. The pathophysiological consequences of obesity for the cardiovascular system have been comprehensively elsewhere [44] but include hypertension, atherosclerosis, impaired cardiac function and arrhythmias. Whether these comorbidities directly translate to increased perioperative risk is not clearly established $[2,45]$. However, that this is potentially a high-risk population is reflected by the attention to this system in the literature retrieved.

Broadly, there were two themes identified: blood pressure monitoring (Table 1) and cardiac output monitoring (Table 2).

Obese patients are more likely to have hypertension than non-obese. It has also been demonstrated that obesity is a risk factor for inadvertent perioperative hypotension (OR of 


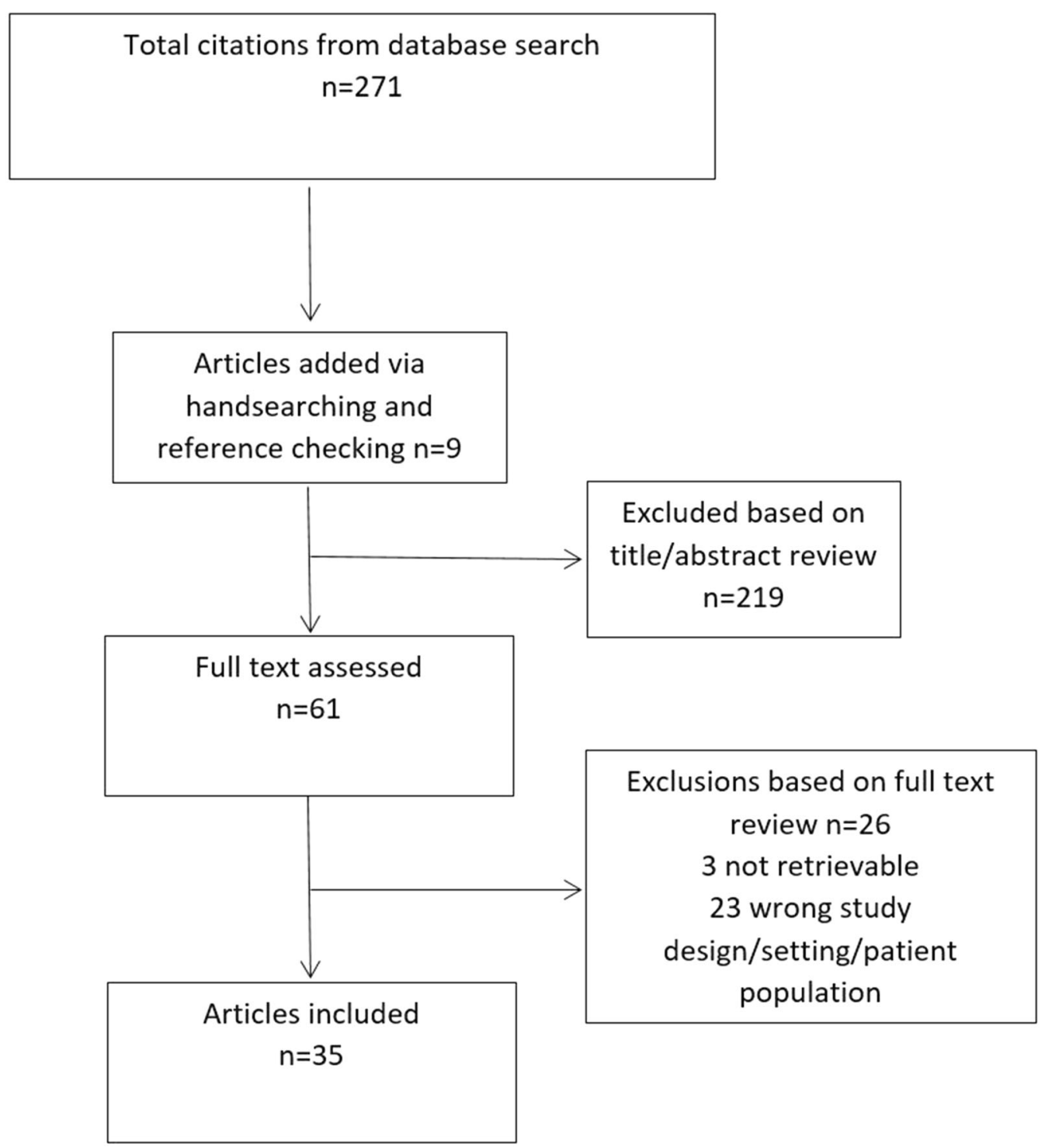

Fig. 1 PRISMA diagram

1.8 for relative hypotension intraoperatively) [46], which itself has been associated with organ damage [47]. A recently published narrative review summarises the difficulties in perioperative blood pressure monitoring in this cohort [48]. The circumference and conical shape of the arm can make non-invasive blood pressure (NIBP) monitoring at the upper arm inaccurate. Longer intervals between NIBP measurements intraoperatively are associated with a four times increased risk of transitioning to hypotension [48]. Continuous measurement may confer more safety in this high-risk cohort.
Traditionally, the mechanism for continuous blood pressure monitoring has been invasive arterial pressure monitoring. Recently, a number of tools for non-invasive continuous measurement have been developed, although the technology was developed and tested in lean patients and has limited evidence base in the obese population [15].

Key considerations are frequency of measurement (intermittent vs. continuous), invasive vs. non-invasive methods, and placement and orientation of blood pressure cuffs. 


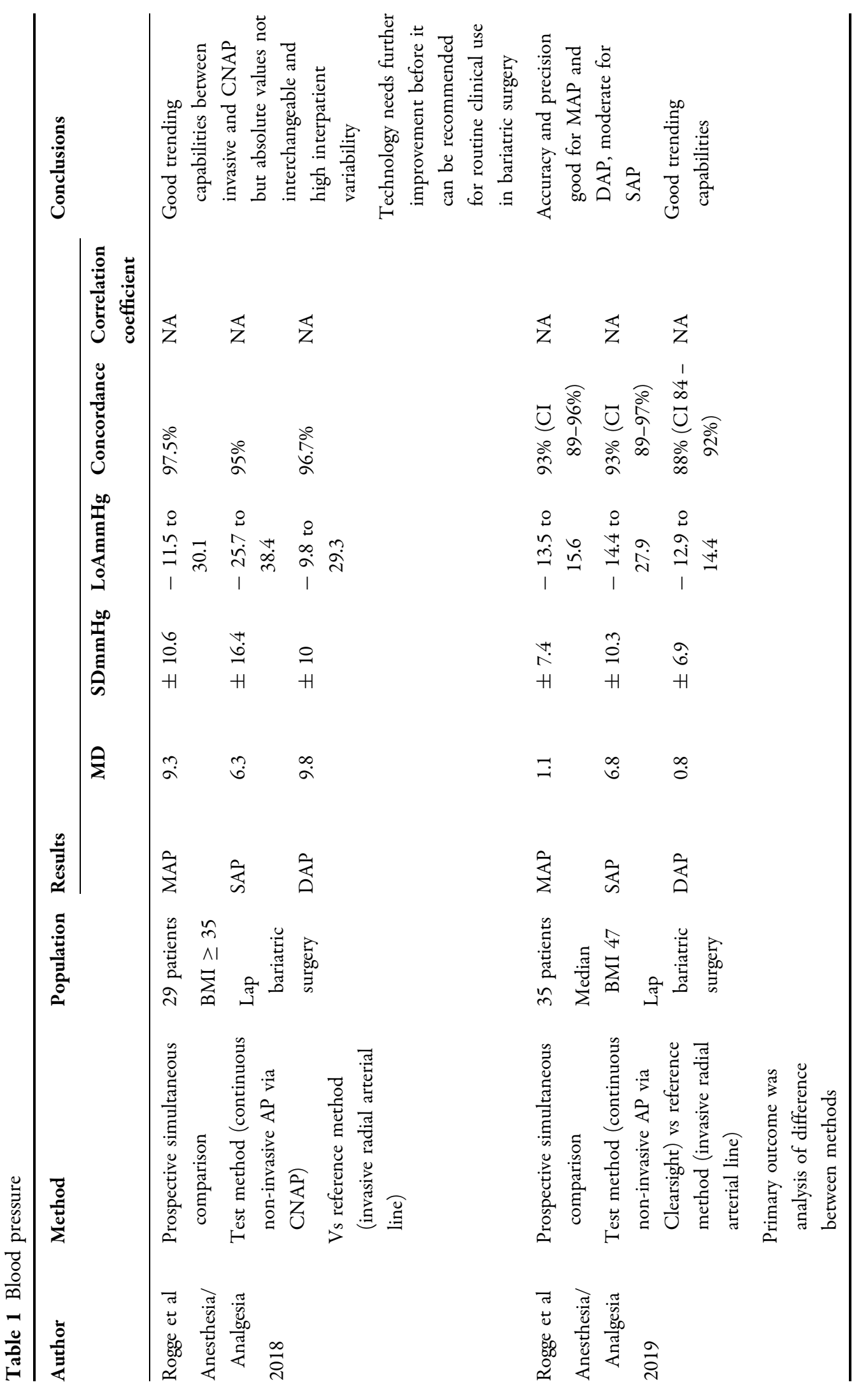




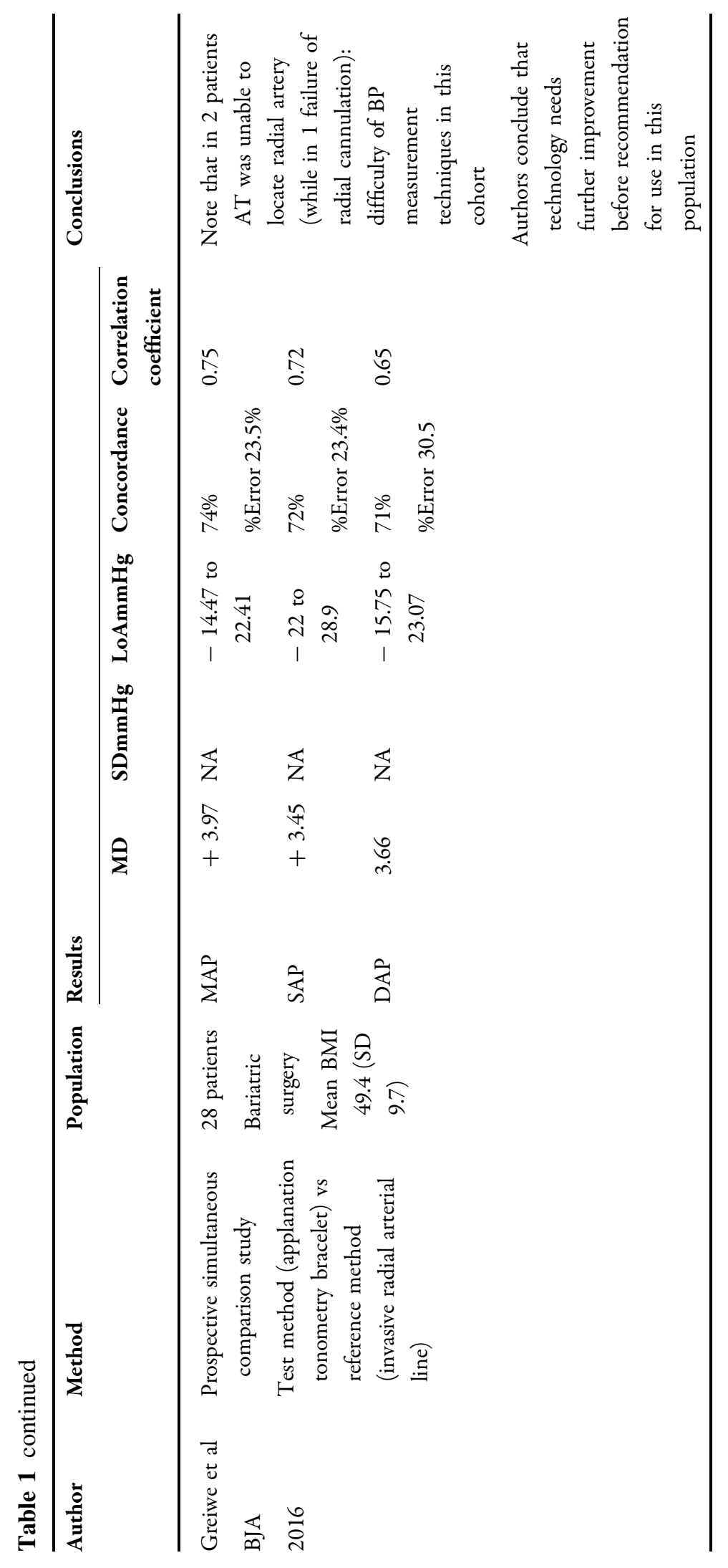




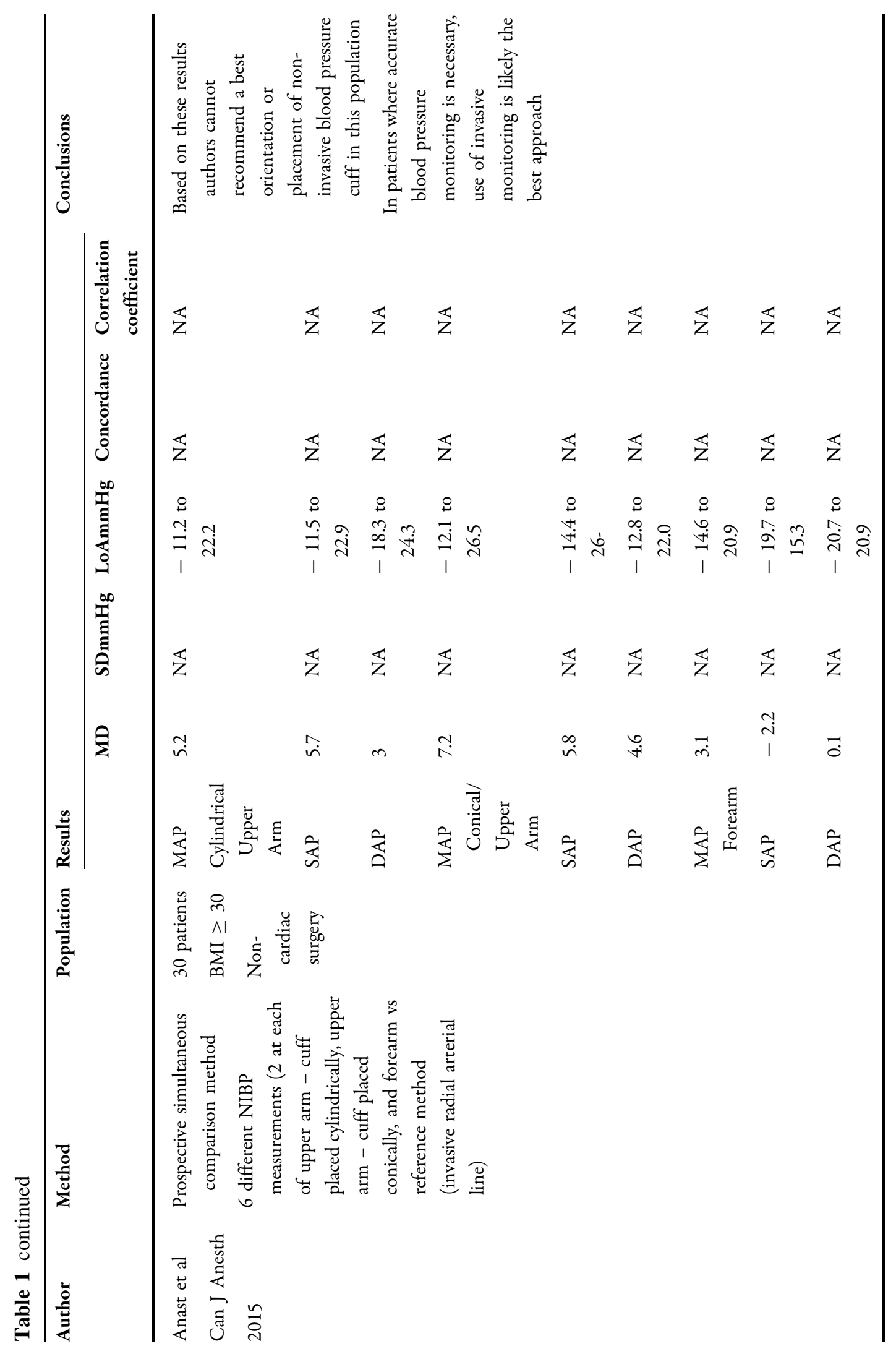




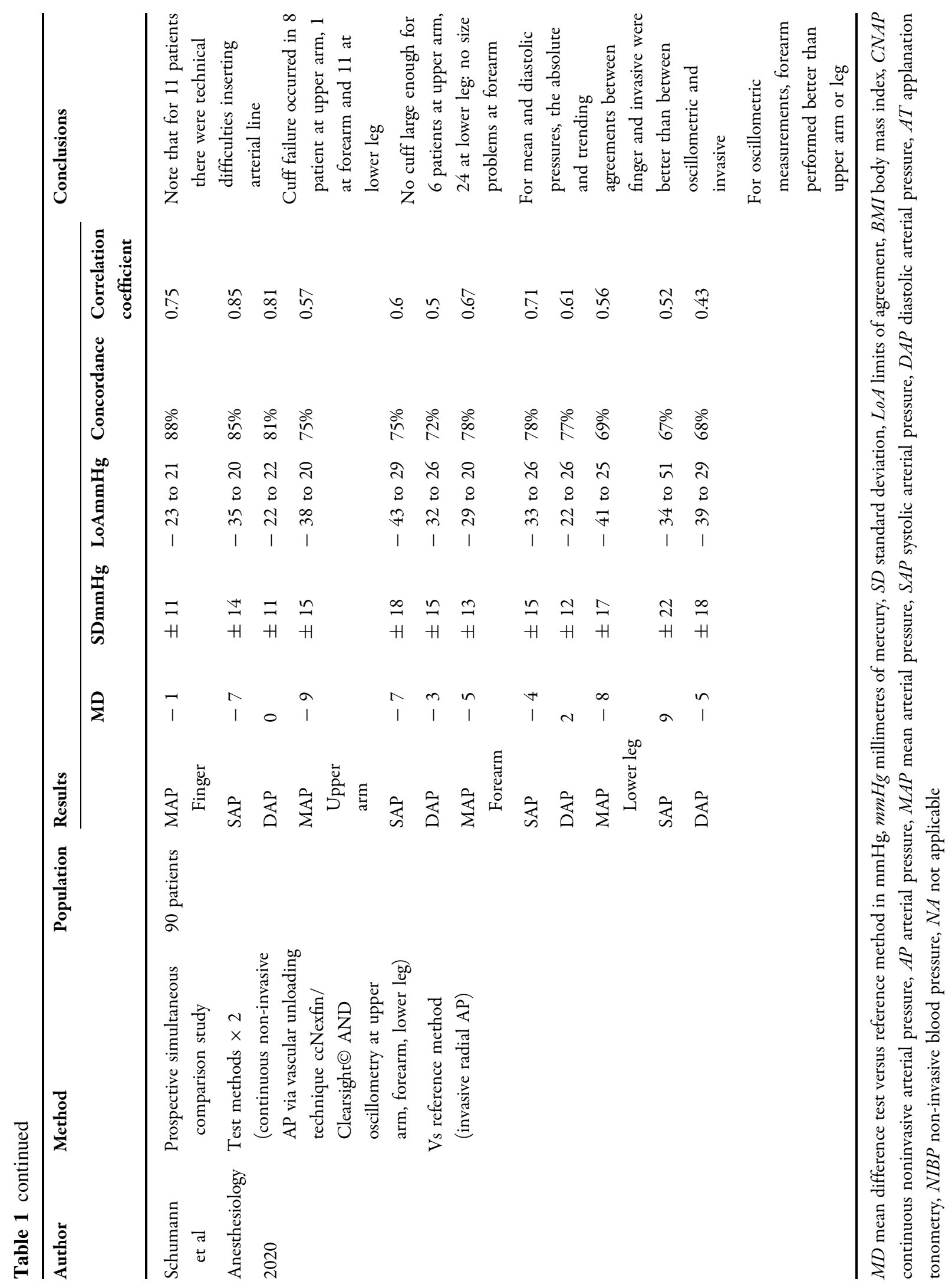




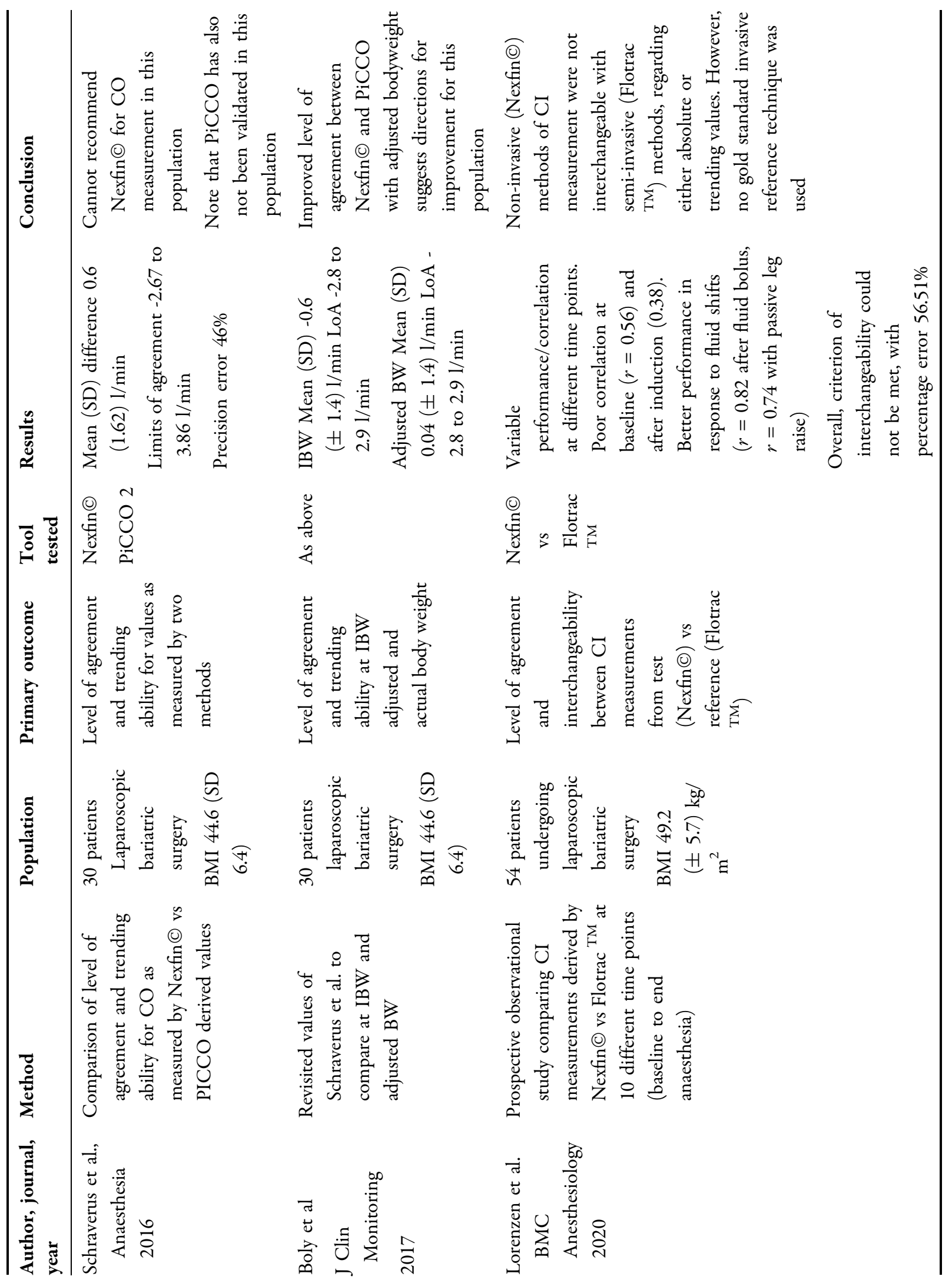




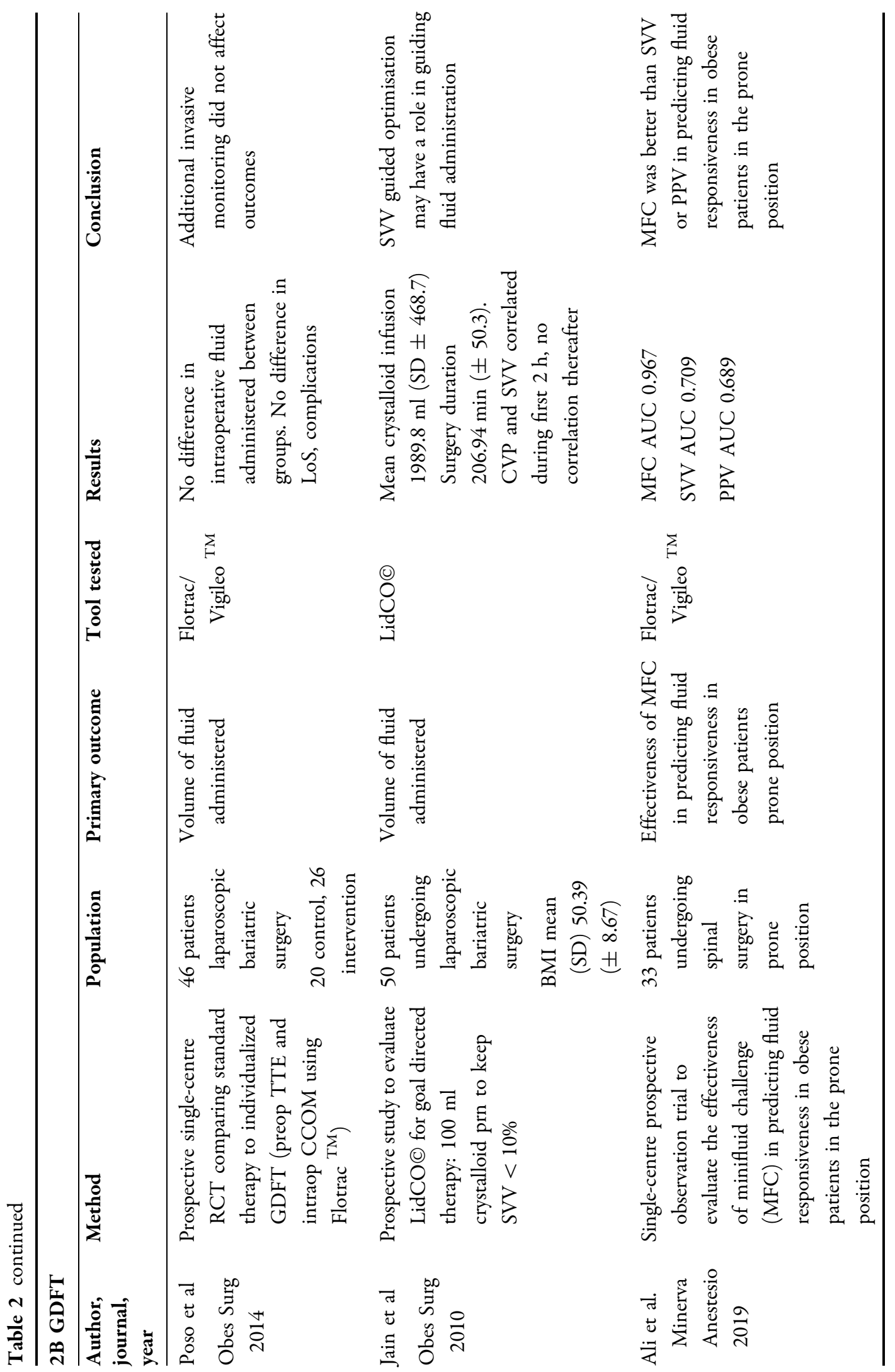




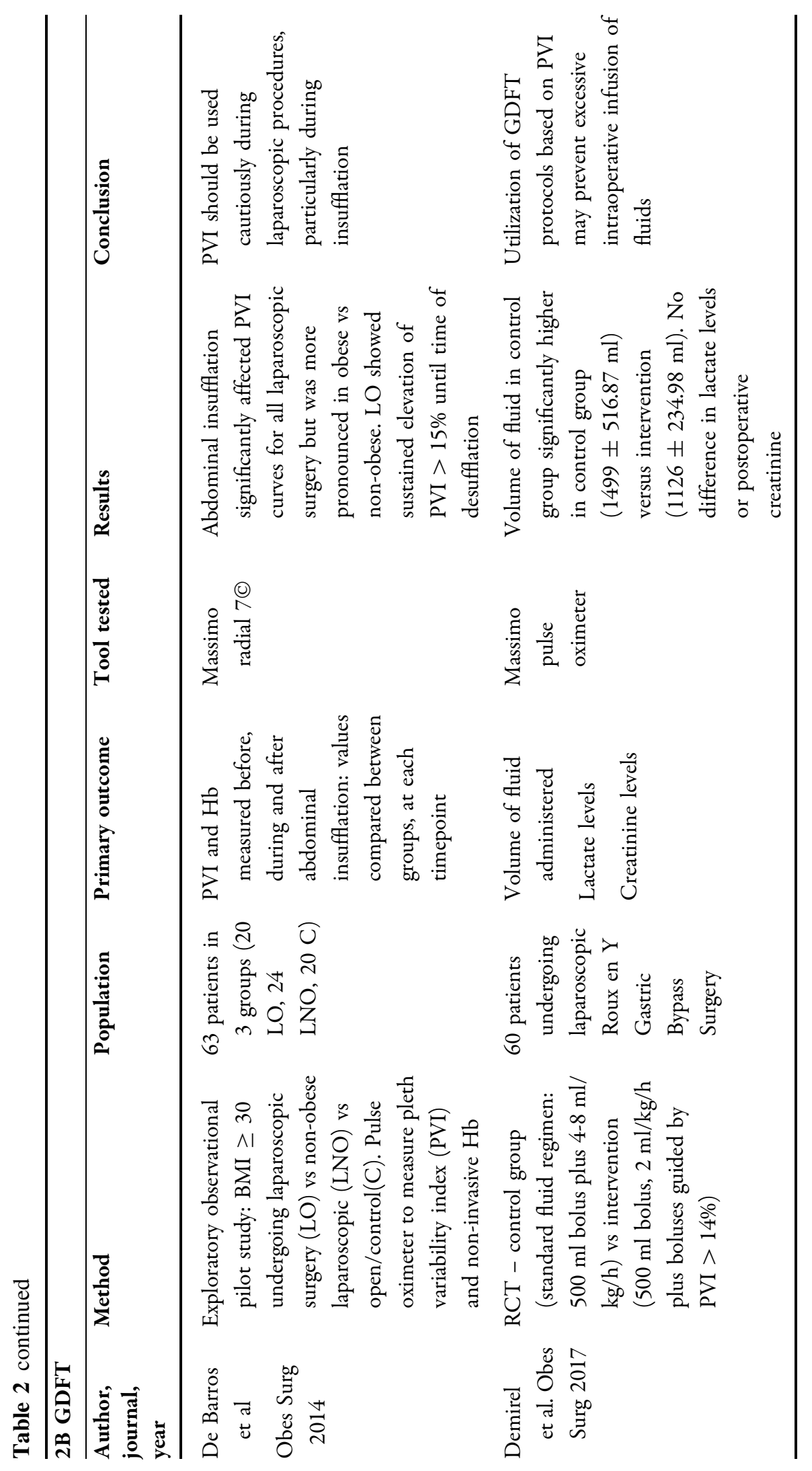




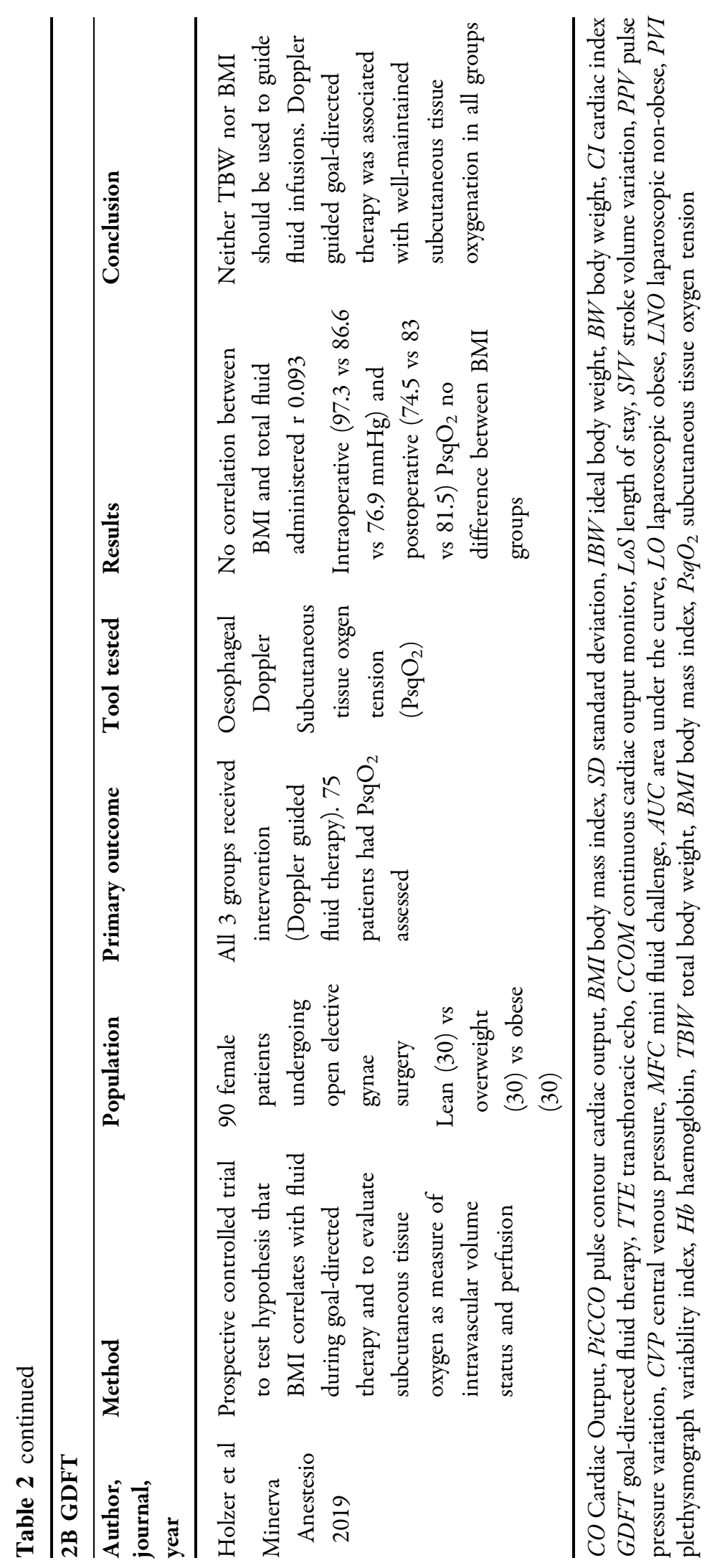




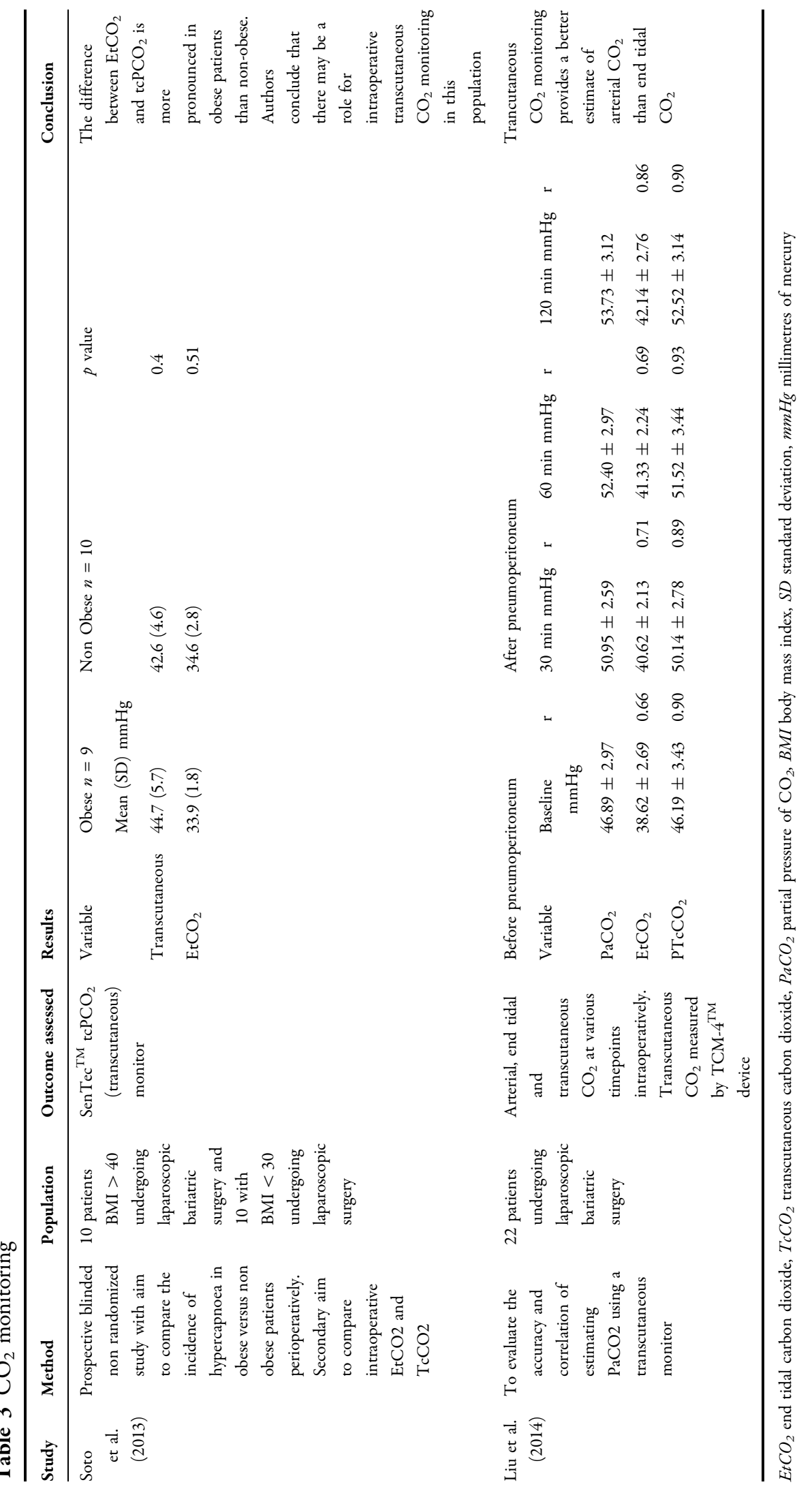


Five studies (Table 1) prospectively and simultaneously compared a reference method (invasive arterial blood pressure monitoring) to a variety of test methods, including continuous non-invasive methods of measurement, and non-invasive blood pressure taken at different sites, and with different cuff orientations.

Continuous non-invasive techniques that were studied included:

1. those based on the volume-clamp method of Penaz, using a finger cuff to measure the change in blood volume of the finger and convert the changes to a continuous waveform using proprietary software [9-11] and

2. those based on applanation tonometry [12], i.e. a sensor bracelet which compresses the radial artery and obtains a continuous arterial pressure signal, processed by an algorithm into a waveform.

While trending capabilities between invasive and non-invasive methods were generally good, the absolute values were not interchangeable, and there was high interpatient variability. The broad conclusion was that this technology needs further refinement and improvement before it can be recommended for use in this cohort, although in the largest comparative study (continuous non-invasive compared to oscillometry at multiple sites and invasive arterial pressure) the finger cuff and invasive methods were comparatively better than the oscillometric versus invasive (concordance $0.75-0.85$ vs. $0.43-0.71$ ) [9].

Two studies examined the optimal placement of the blood pressure cuff for non-invasive measurements. In one [9], the forearm performed better than either the upper arm (failed in $15.5 \%$ ) or lower leg (failed in 39\%). However, in the other, the authors [13] were unable to recommend a best orientation or placement of the blood pressure cuff and suggest that in patients where accurate blood pressure monitoring is necessary, use of invasive monitoring is likely the best approach. The limitations of invasive arterial pressure monitoring in this cohort are also demonstrated: difficulty siting an arterial line was reported in $12 \%$ of patients in one study [9], indicating the challenges even of the reference standard.
Nine studies considered techniques for monitoring cardiac output intraoperatively, three of which compared two methods of cardiac output monitoring (Table 2a) and six of which considered individualised goal-directed fluid therapy (Table 2b).

Cardiac output monitoring methods can be categorised according to invasiveness (invasive, minimally invasive, and non-invasive) and according to calibration (external calibration vs. uncalibrated methods) [49]. The summary conclusion from the three comparative studies is that there is limited agreement between derived measures of cardiac output and that values were not interchangeable. Overall, there was a guarded applicability of the tested methods to the obese population (albeit a suggestion that inputting adjusted body weight into the algorithm might improve the performance [15]).

Goal-directed fluid therapy (GDFT) is the optimization of tissue perfusion by targeted fluid management. GDFT protocols have been demonstrated to reduce morbidity and length of stay in high-risk surgeries [50]. Excessive perioperative fluid administration has an established adverse effect: the consequence of administration of each extra litre of fluid intraoperatively is an estimated 32\% increase in postoperative complications [51]. Meanwhile, a large trial (to which $\mathrm{BMI} \geq 35$ was an inclusion criterion) comparing restrictive (designed to produce net zero fluid balance) to liberal perioperative fluid regimes demonstrated an increased risk of acute kidney injury with restrictive regime [52]. This is of particular concern to the obese surgical patient, in whom the risk of rhabdomyolysis [53] and acute kidney injury [54] is already elevated. Inclusion of non-invasive, dynamic indicators to guide GDFT is suggested for this population [7].

Dynamic indices of fluid responsiveness include respiratory variations in the pulse oximeter (plethysmograph variability indices, PVI) or in the arterial pulse pressure (stroke volume variation [SVV] or pulse pressure variation [PPV]). Six studies (Table 2b) explored the role of goal-directed fluid therapy using diverse expressions of these techniques. The results in this population were somewhat equivocal. In one study, mini fluid challenge was superior to 


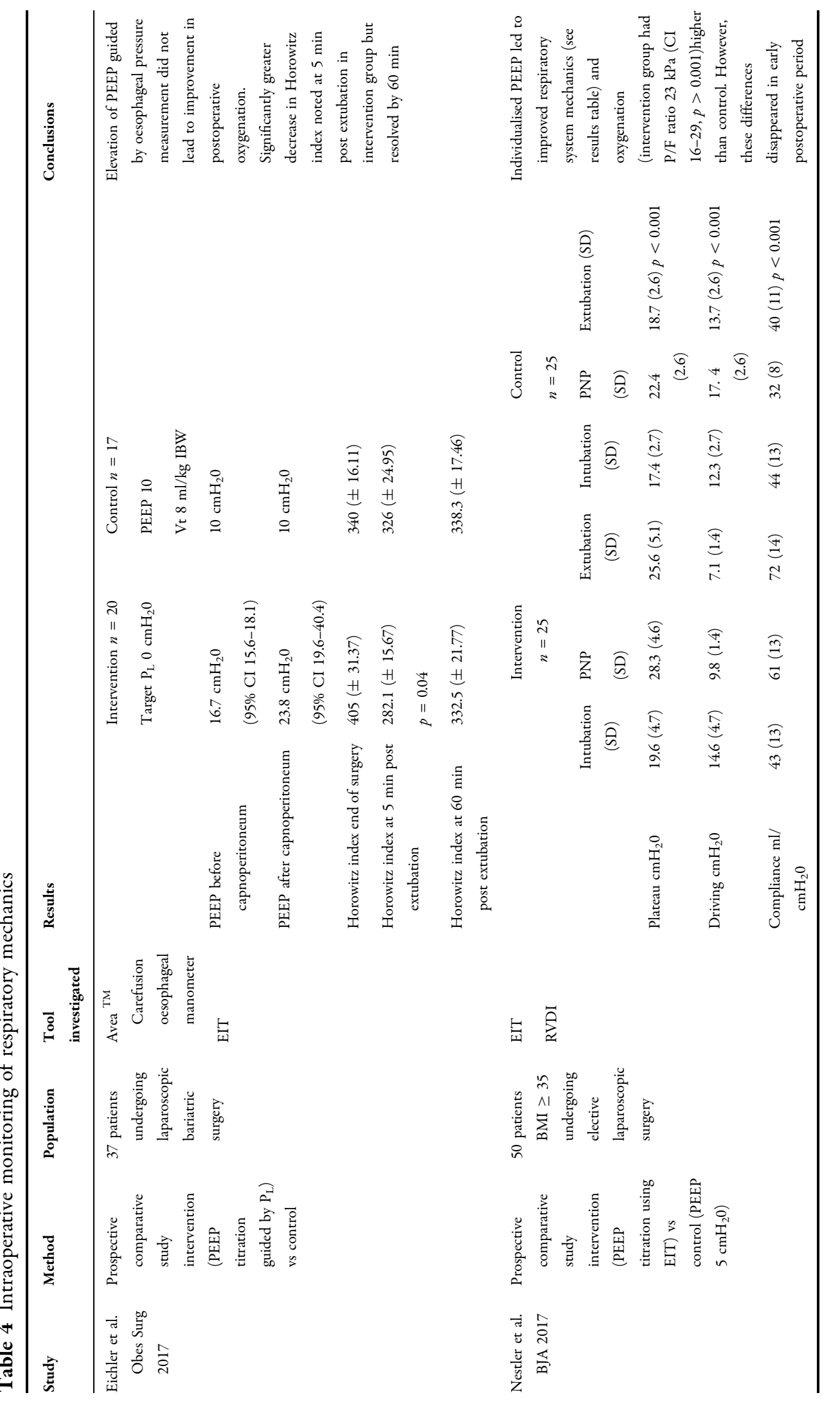




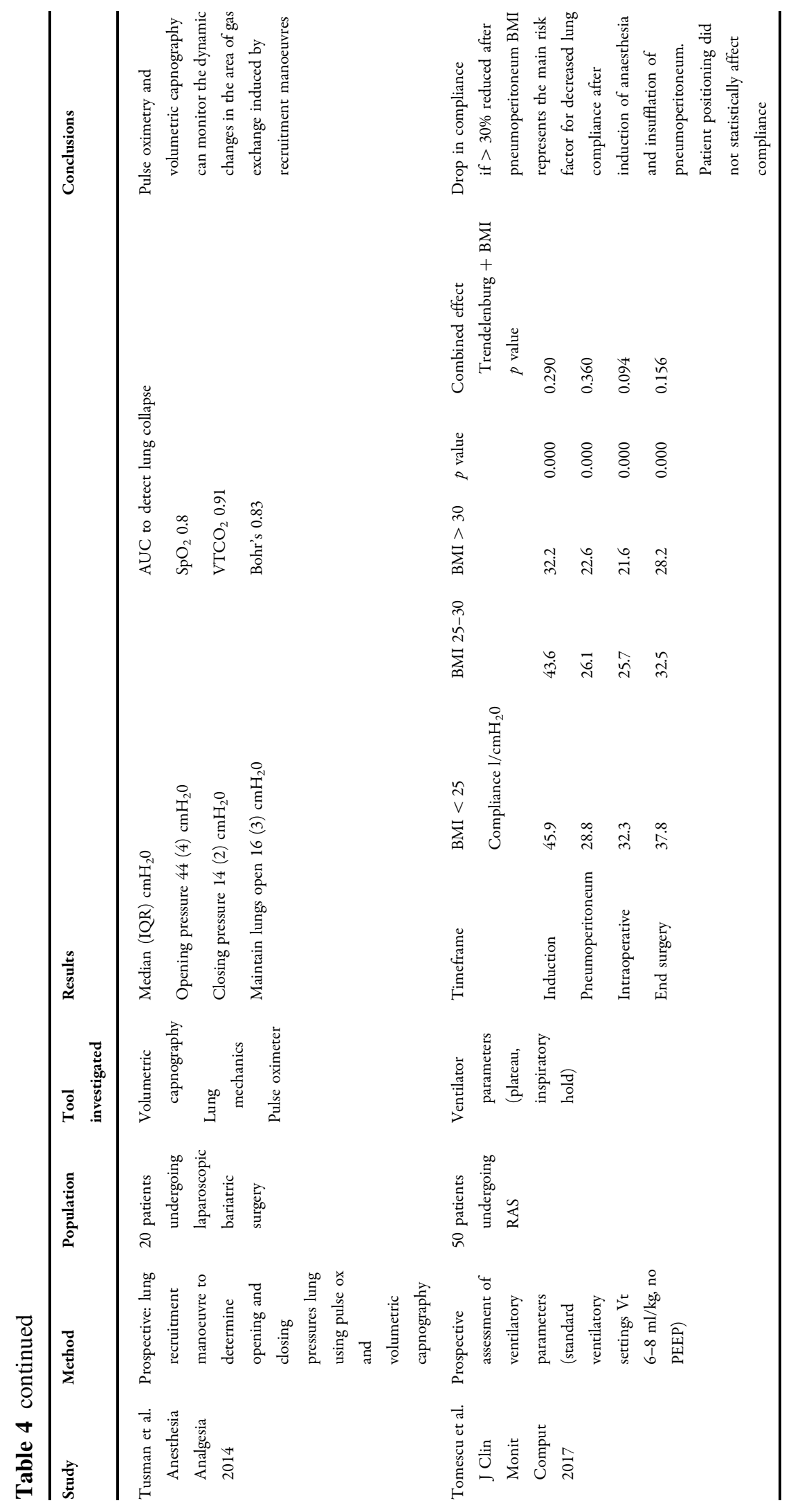




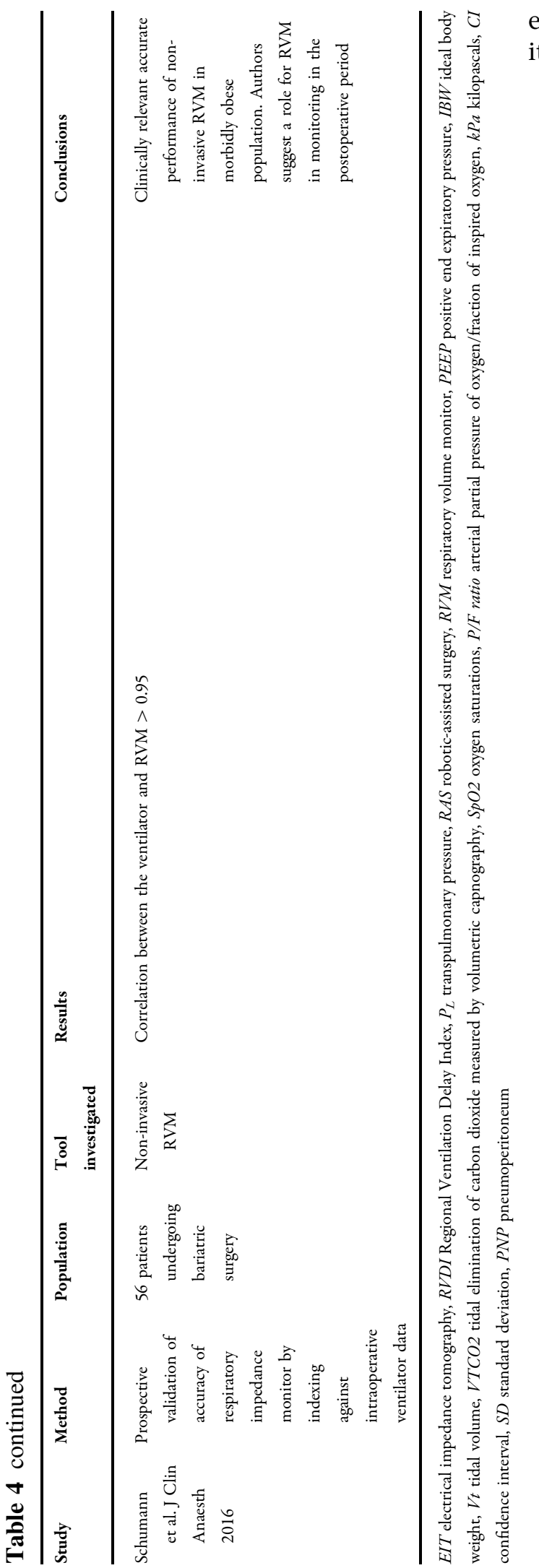

e- $\quad$ her SVV or PPV in predicting fluid responsiveit- ness (AUC 0.967 vs. 0.709 and 0.689 respectively) [18]. Another found no additional benefit to using semi-invasive cardiac output monitoring regarding intraoperative fluid administered, postoperative complications, or length of stay [16]. Finally, there were conflicting results regarding the non-invasive PVI in this population-while in one study, PVI-guided fluid resulted in a lower volume overall with no detrimental consequences of increases in lactate or postoperative creatinine [21], another showed the unreliability of PVI in laparoscopic procedures [19]. Although endorsed in clinical practice guidelines [7], it is difficult to draw conclusions about the optimal technique to guide fluid administration.

\section{Respiratory}

The respiratory system is a focus of the existing guidelines on perioperative management of the obese patient. Particular emphasis is placed on the identification and preoperative treatment of sleep disordered breathing which confers increased risk at every point of the perioperative pathway, including increased incidence of difficult intubation and increased risk of postoperative pulmonary complications (PPCs).

Respiratory mechanics are significantly altered in obesity and may be further compromised by anaesthesia and surgical access. Increased intra-abdominal pressure combined with decreased total lung capacity, vital capacity, and functional residual capacity lead to atelectasis. Decreased pulmonary compliance intraoperatively, particularly in laparoscopic surgery, leads to higher transthoracic and transpulmonary pressures, increasing the risk of ventilator-induced lung injury. The contribution to surgical mortality attributable to postoperative lung injury is estimated at 19\% [55].

PPCs are of particular relevance to the obese population. A subgroup analysis of obese patients recruited within a multicentre international trial found an overall incidence of $10.4 \%$ PPCs at postoperative day 5 , which increased to $18.5 \%$ for Class 3 obesity. PPCs were associated with increased length of 


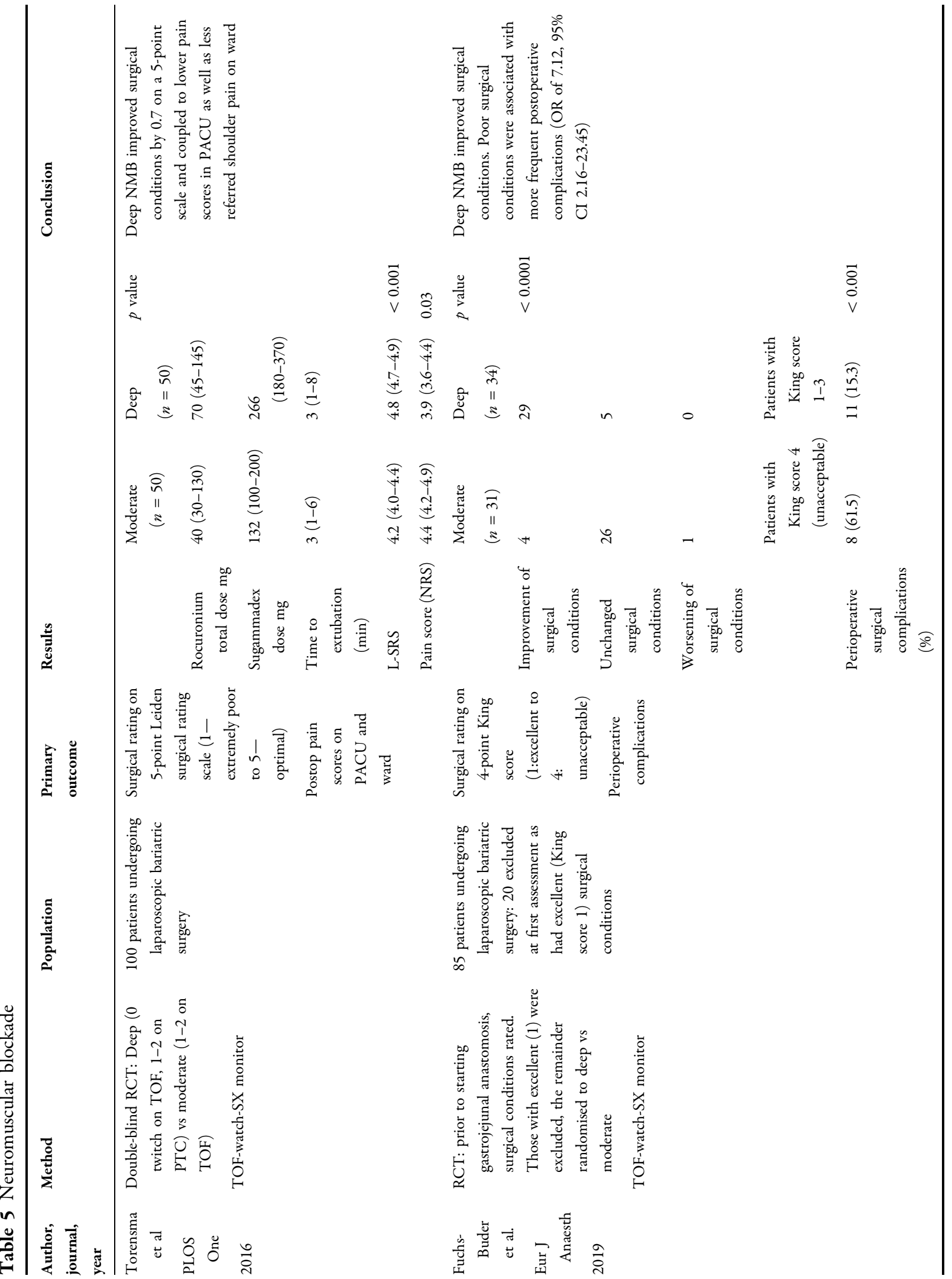




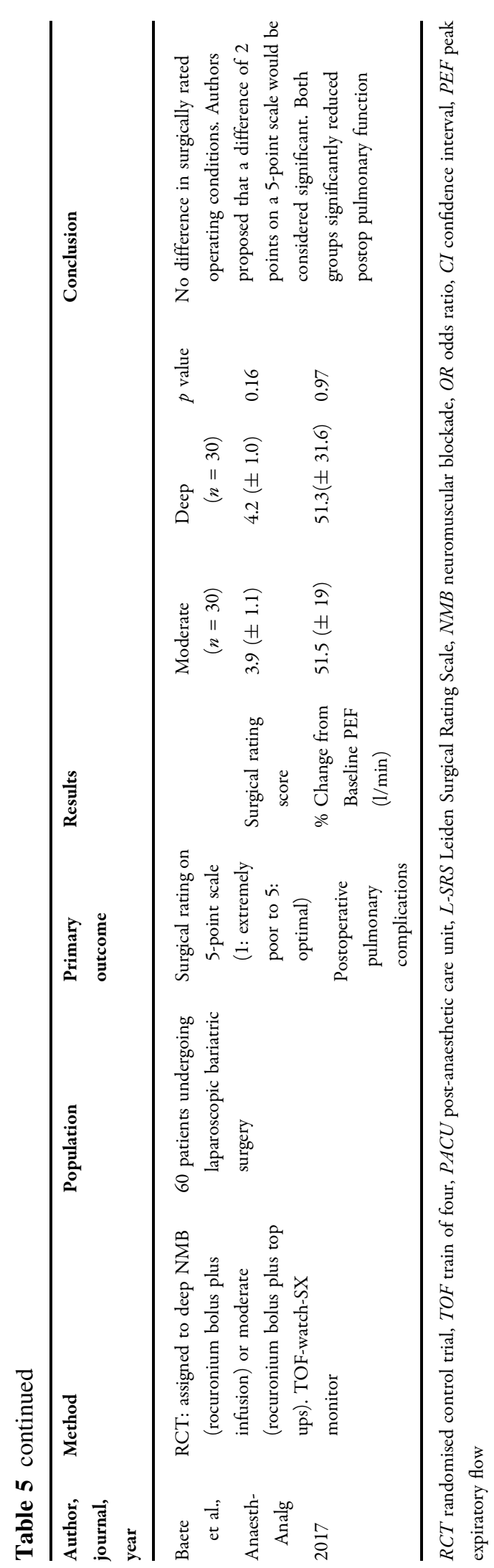

hospital stay [56]. Furthermore, a trial of high PEEP and recruitment manoeuvres vs. low PEEP in almost 2000 obese $(\mathrm{BMI} \geq 35)$ patients undergoing noncardiac, non-neurological surgery demonstrated an even higher rate of PPCs at day 5 , at $21.3 \%$ in the intervention and $23.6 \%$ in the control group [57].

Broadly, two themes were identified: carbon dioxide $\left(\mathrm{CO}_{2}\right)$ monitoring and quantification of respiratory mechanics.

Two studies (Table 3) looked at the intraoperative use of transcutaneous $\mathrm{CO}_{2}$ monitors. Capnography has limitations in obese patients because of a greater expired $\mathrm{CO}_{2}$ to arterial $\mathrm{CO}_{2}$ gradient than in the non-obese due to increased ventilation/perfusion mismatching [23, 58]. Transcutaneous $\mathrm{CO}_{2}$ monitors use a variant of the Severinghaus-type $\mathrm{CO}_{2}$ electrodes, where warmed skin allows $\mathrm{CO}_{2}$ to diffuse into an electrolyte solution. This has previously been shown to be more accurate in the obese, with a difference of up to $1 \mathrm{kPa}$ between methods [58]. Liu et al. [24] similarly concluded that transcutaneous $\mathrm{CO}_{2}$ provided a better estimate of arterial $\mathrm{CO}_{2}$ than end tidal values (correlation coefficient 0.9 versus 0.66 for baseline values).

There may be a role for intraoperative transcutaneous $\mathrm{CO}_{2}$ monitoring in this cohort in addition to (rather than in place of) capnography. Furthermore (and as was the primary focus of one study [23]), transcutaneous $\mathrm{CO}_{2}$ monitoring may facilitate safety in the postoperative period.

An increased BMI constitutes the major risk factor for decreased lung compliance during robotic surgery [28]. Adjustment of intraoperative ventilatory parameters has been proposed as a strategy to overcome the adverse respiratory mechanics experienced by the obese patient, particularly undergoing laparoscopic surgery. An excellent narrative review has been published on intraoperative mechanical ventilation strategies, with a focus on specific populations including the obese [56]. We identified several heterogeneous studies which test this approach (Table 4). One study examined the role of volumetric capnography (AUC 0.91) and pulse oximetry (AUC 0.8 ) in objectively assessing the impact of a recruitment manoeuvre. Notably, a median pressure of $16 \mathrm{cmH}_{2} \mathrm{O}$ was required to 


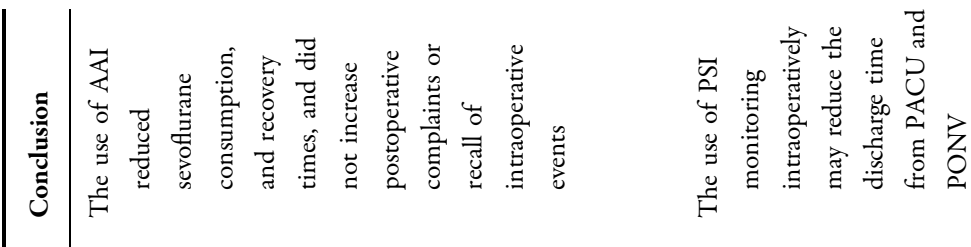

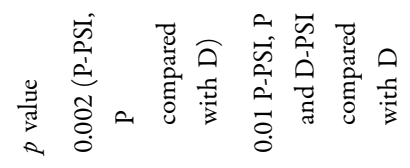

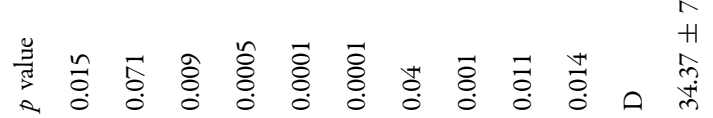

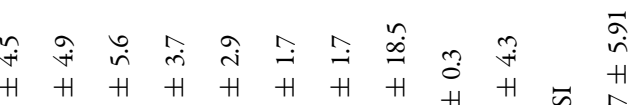

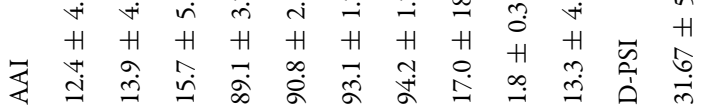

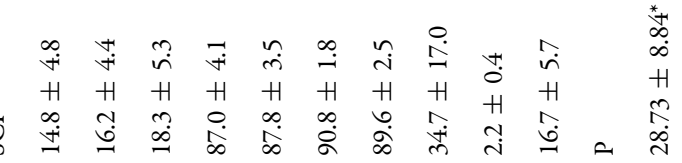

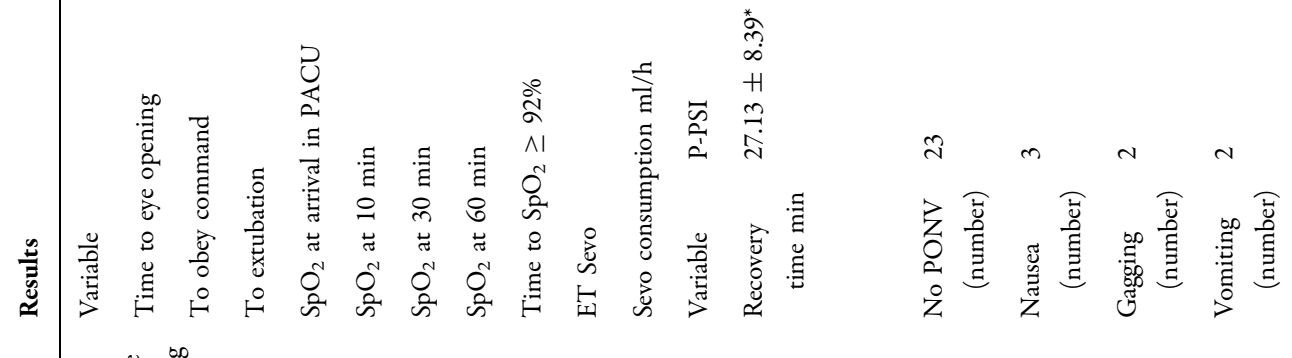

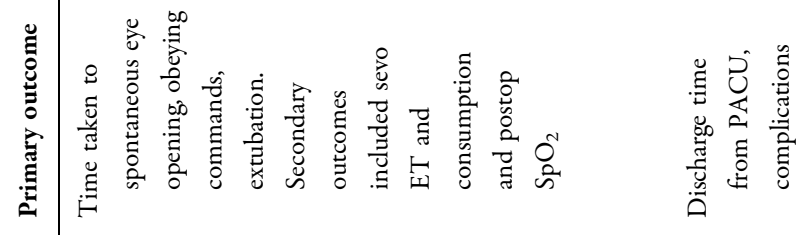

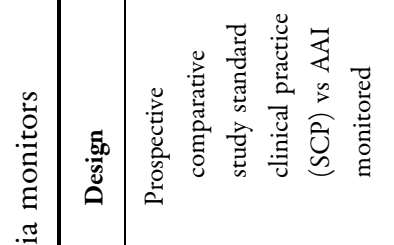

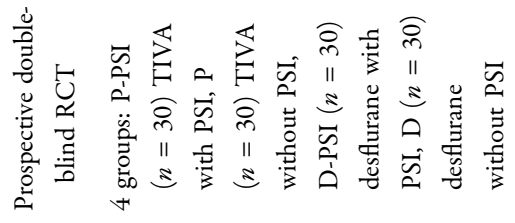

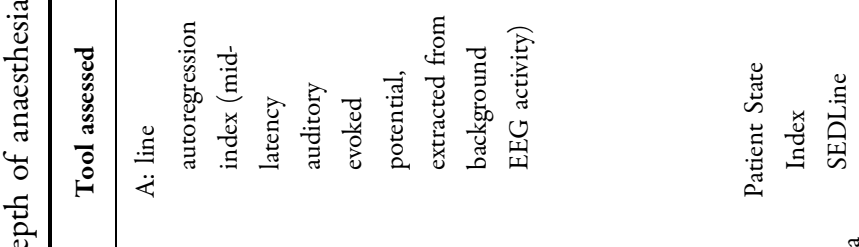

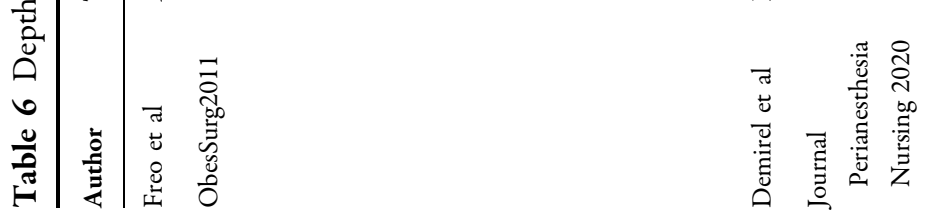




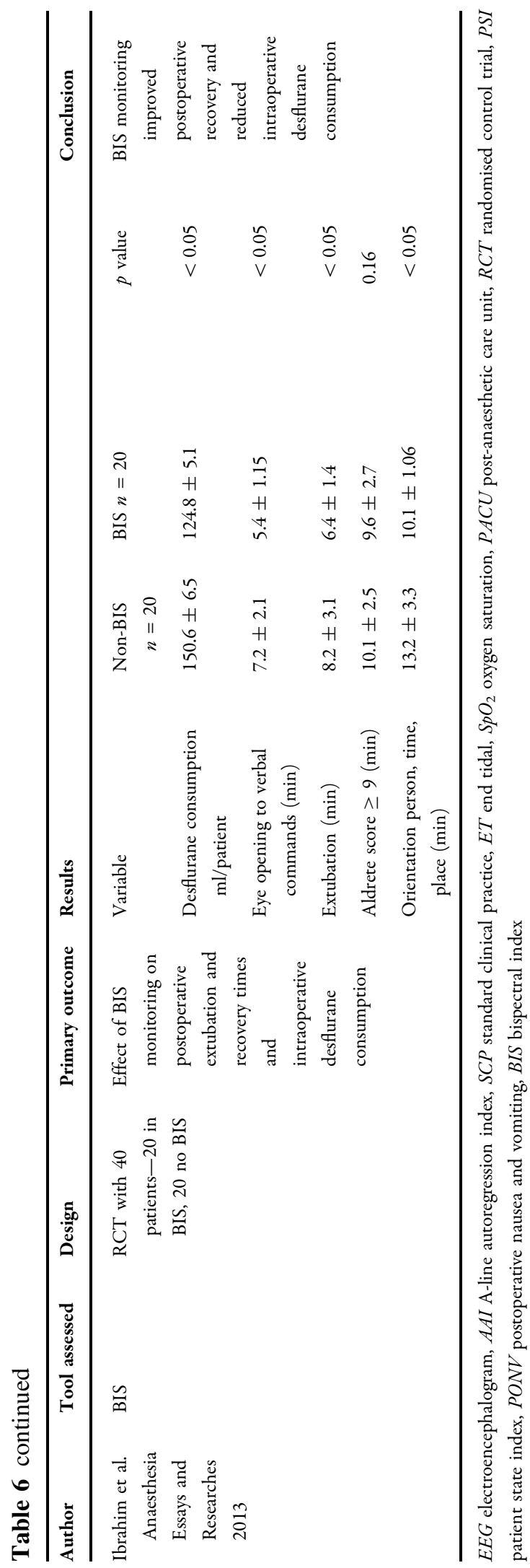

keep the lungs "open" [27]. Two studies explored the use of electrical impedance tomography (EIT) to facilitate an individualised titration of PEEP to test the hypothesis that this would offset the atelectasis and consequent decrease in arterial oxygenation that occur in obese patients undergoing general anaesthesia $[25,26]$. While both showed an intraoperative improvement in both mechanics and oxygenation, the differences were not sustained into the postoperative oxygenation period.

There is some evidence to suggest that individually titrated PEEP may mitigate the adverse respiratory mechanics at which the obese patient is at higher risk. However, none of the studies demonstrated an improvement into the immediate postoperative period. It is difficult to draw conclusions about the utility of additional intraoperative respiratory monitoring in this population, although in the postoperative period a non-invasive respiratory volume monitor may have a role [29].

\section{Neuromuscular}

Four key themes emerged: neuromuscular blockade monitoring, depth of anaesthesia monitoring, cerebral perfusion, and assessment of pain.

Peripheral nerve stimulators form part of the minimum standards for anaesthetic monitoring, where muscle relaxants have been used [8]. The National Audit Project (NAP) 5 audit into accidental awareness under anaesthesia (AAGA) concluded that the combination of using muscle relaxants, without monitoring their effect, and without reversing them, incurred a risk for AAGA that was roughly 16 times greater when muscle relaxants were used versus none. So strongly was neuromuscular blockade implicated in AAGA that the authors suggest a subtitle of "unintended awareness under neuromuscular block". Over three times as many obese patients experienced AAGA as nonobese [59]. Furthermore, the incidence of postoperative residual blockade is higher in the obese patient [60]. Consensus guidelines indicate that where muscle relaxants are used in this 
Table 7 Near infrared spectroscopy

\begin{tabular}{|c|c|c|c|c|c|c|}
\hline Author & Tool assessed & Design & $\begin{array}{l}\text { Primary } \\
\text { outcome }\end{array}$ & Results & & Conclusion \\
\hline $\begin{array}{l}\text { Ruzman } \\
\text { et al } \\
\text { Surg } \\
\text { Laparosc } \\
\text { Endos } \\
\text { Percut } \\
\text { Tech } \\
2017\end{array}$ & $\begin{array}{l}\text { Near infrared } \\
\text { spectroscopy } \\
\text { to measure } \\
\text { regional } \\
\text { cerebral } \\
\text { oxygen } \\
\text { saturations } \\
\left(\mathrm{rSO}_{2}\right)\end{array}$ & $\begin{array}{l}\text { Prospective, all } \\
\text { patients } \\
\text { undergoing } \\
\text { laparoscopic } \\
\text { cholecystectomy. } \\
\text { Analysed for } \\
\text { association } \\
\text { between patient } \\
\text { characteristics and } \\
\text { critical (drop of } \\
20 \% \text { from baseline } \\
\text { value) } \mathrm{rSO}_{2} \text { level }\end{array}$ & & $\begin{array}{l}\text { Induction supine for } \\
\text { BMI }>30 \text { Right } \\
\text { Pneumoperitoneum } \\
\text { right for } \\
\text { BMI }>30 \\
\text { Pneumoperitoneum } \\
\text { left for BMI }>30 \\
\text { Reverse } \\
\text { Trendelenburg } \\
\text { right for } \\
\text { BMI }>30 \\
\text { Reverse } \\
\text { Trendelenburg } \\
\text { left for BMI }>30\end{array}$ & $\begin{array}{l}p=0.045 \\
p=0.027 \\
p=0.045 \\
p=0.012\end{array}$ & $\begin{array}{l}\text { Significantly greater } \\
\text { declines in } \mathrm{rSO}_{2} \\
\text { were noted in } \\
\text { elderly } \\
(\geq 65 \text { years) and } \\
\text { obese (BMI } \geq 30) \\
\text { in this cohort } \\
\text { during surgery. No } \\
\text { association } \\
\text { between these } \\
\text { factors and } \\
\text { baseline rSO } \\
\text { Authors conclude } \\
\text { that non-invasive } \\
\text { measures of } \\
\text { cerebral } \\
\text { oxygenation could } \\
\text { be helpful in } \\
\text { "higher risk" } \\
\text { patients including } \\
\text { elderly and obese }\end{array}$ \\
\hline
\end{tabular}

NIRS near-infrared spectroscopy, $r \mathrm{SO}_{2}$ regional cerebral oxygen saturations, $\mathrm{BMI}$ body mass index

population, neuromuscular blockade should be assessed and reversed prior to extubation [4].

The second aspect to be considered is deep (defined as no twitches on train of four [TOF] or post-tetanic count [PTC] of one or two) versus moderate (two twitches on TOF) blockade. Recent guidelines concluded that there was insufficient data to make recommendations about deep versus moderate blockade for obese patients undergoing laparoscopic surgery [61]. Despite this, deep blockade is, in practice, more commonly used in this population: a retrospective review of 88,000 general anaesthetics in which muscle relaxants were used found that a higher BMI class was the major patient-specific factor associated with deep blockade [62]. Three studies looked at deep versus moderate neuromuscular blockade (Table 5). Two studies found that deep neuromuscular blockade improved surgeon-reported operator conditions during laparoscopic bariatric surgery, with a consequent reduction in perioperative complications [36] and pain [35]. There was no increase in time to extubation with deep blockade, although dose of reversal was higher. A third study $(n=60)$ [37], found no difference in operating conditions with deep blockade and no difference in length of surgery. A secondary outcome was postoperative pulmonary function tests: both groups demonstrated a marked decline in function (50\% reduction in peak expiratory flow, FEV1 and FVC) with no difference between deep and moderate blockade. As with all patients, where neuromuscular blockade is used, postoperative reversal should be confirmed before the end of the case (to confirm return of "motor capacity" [59]). This is a central tenet of anaesthesia practice and is not solely 


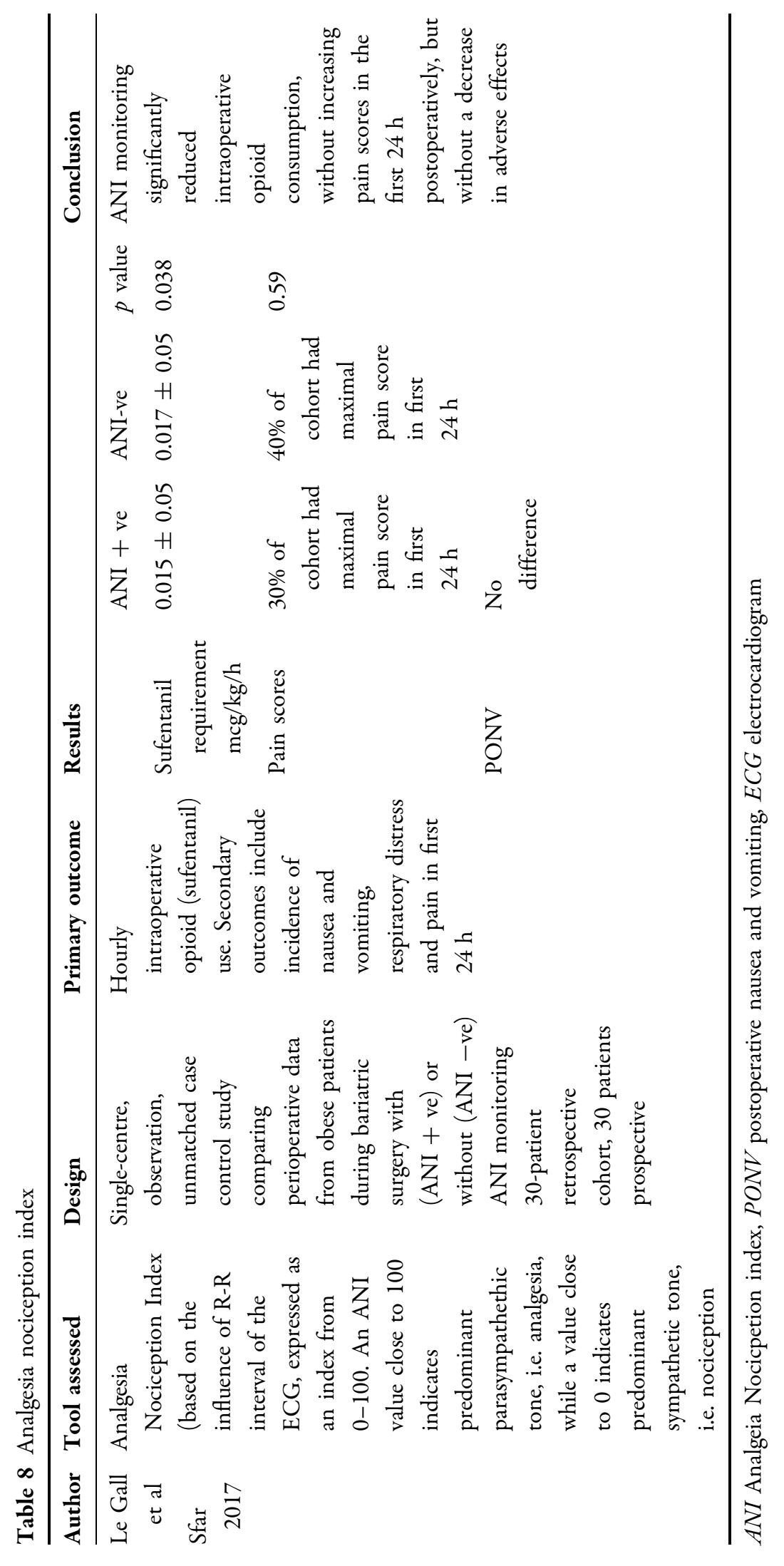




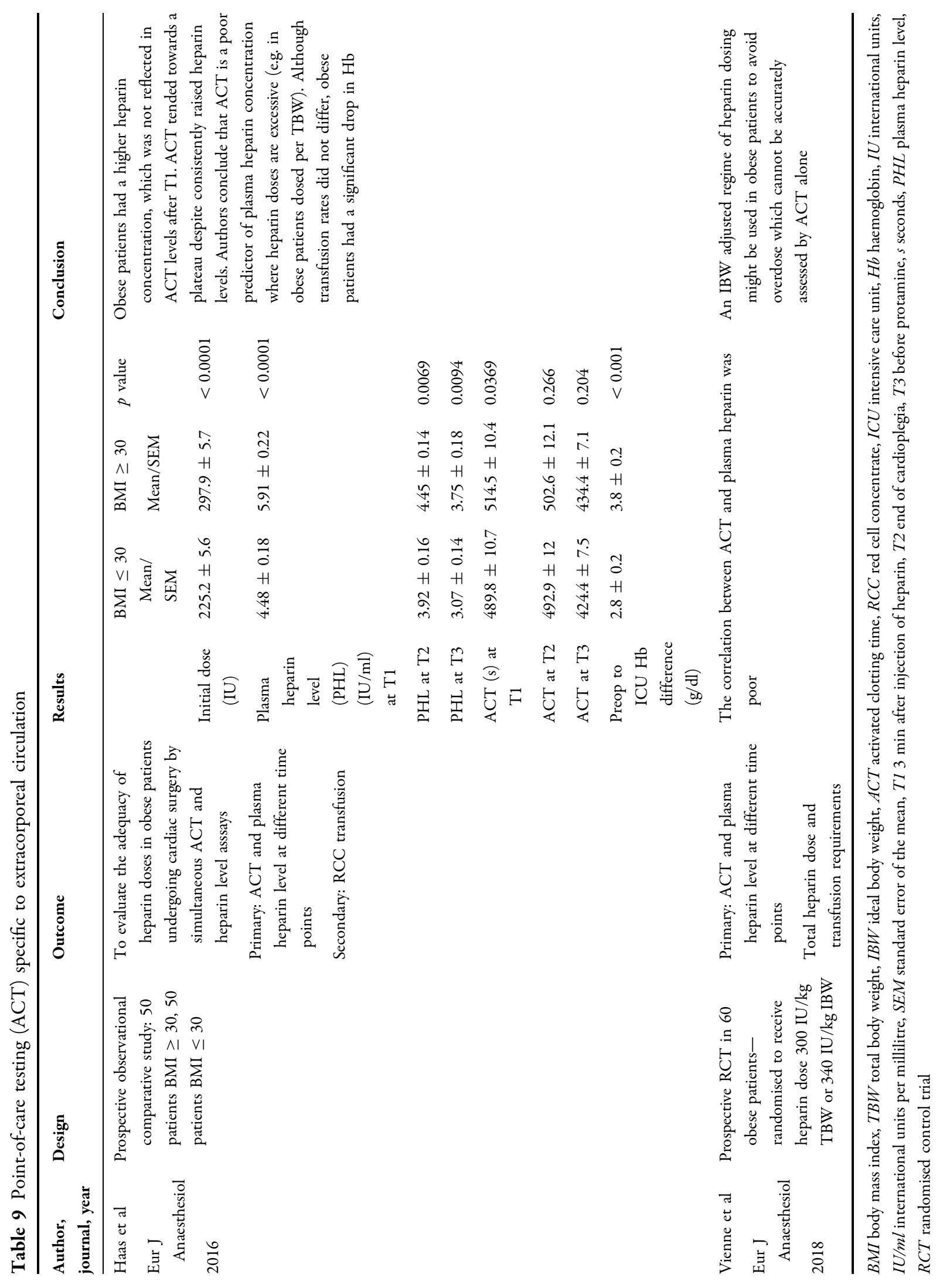


applicable to the obese patient. International studies of practice, however, have suggested that this may be a neglected area of practice [63]. Meticulous attention to this standard aspect of anaesthetic management is important to mitigate against postoperative complications in this "at risk" extubation group [60].

There may be a role for deep blockade to improve surgical conditions, in which case continuous neuromuscular monitoring aiming for a post-tetanic count of 1-2 is suggested.

Although not specified in the minimum standards of anaesthesia monitoring guideline [8], depth of anaesthesia (DOA) monitoring arguably constitutes a key aspect of intraoperative monitoring of the obese patient $[4,5]$. Three studies tested the depth of the anaesthesia monitor (see Table 6). Though each study tested a different monitor, all three demonstrated a reduction in recovery times. Consumption of vapour was reduced when DOA was utilised, with no increase in recall of intraoperative events. When compared to standard clinical practice, intraoperative DOA use was also associated with improved postoperative $\mathrm{SpO}_{2}$. Given the importance of postoperative pulmonary complications in this cohort, this may constitute additional justification for their use. No studies compared one DOA monitor to another. There is no evidence to direct whether one DOA monitor is superior to another.

Near-infrared spectroscopy is a non-invasive technique, which quantifies regional cerebral saturation $\left(\mathrm{rSO}_{2}\right)$ as a surrogated for cerebral perfusion. Reduction in cerebral oxygenation has been associated with increased risk of neurocognitive disorders postoperatively. In a "lowrisk" cohort of ASA 1 and 2 patients, a significant proportion (>20\%) experienced a critical decline in cerebral saturations (Table 7) [34]. Obese patients were at statistically increased risk of experiencing this (perhaps due to impaired compensatory mechanisms in response to pneumoperitoneum). Intraoperatively, a protocol was implemented to act on the drop in $\mathrm{rSO}_{2}$ and no postoperative neurocognitive assessment was conducted so the impact of the decline cannot be ascertained.

Multimodal analgesic strategies are prioritised in the obese patient to reduce the use of opioids perioperatively and thereby avoid the respiratory depression and nausea and vomiting which are of particular import in this context. The analgesia nociception index (ANI) has been proposed for the objective evaluation of pain perioperatively, providing an index value from 0 (predominant sympathetic tone, high level of stress, nociception) to 100 (predominant parasympathetic tone, low level of stress, analgesia). One study was retrieved (see Table 8) which found that opioid consumption was significantly reduced in the first $24 \mathrm{~h}$ postoperatively when ANI was used. However, this did not associate with a decrease in adverse effects. This is a novel technique, with limited evidence in the literature (our search retrieved only one additional case report) [64] but given the general preference for opioid-sparing techniques perioperatively, and the particular benefits this might have for the obese patient, this is a topic in which the evidence base will likely expand.

\section{Endocrine/Metabolic}

Adults with $\mathrm{BMI} \geq 40$ are 7 times more likely to have diabetes compared those with normal weight [65]. Meticulous attention to perioperative glycaemic levels is crucial as poor control is associated with increased perioperative complications. This is of particular importance to the patient undergoing bariatric surgery, as gastric bypass surgery results in glycaemic instability, with a surgical stress response counterpointed by a rapid reduction in insulin requirements [66]. One study was retrieved which explored continuous glucose monitoring in bariatric patients undergoing sleeve gastrectomy (LSG) and Roux-en-Y bypass(LRYGB) [41]. Throughout the perioperative period, from $24 \mathrm{~h}$ prior to surgery for 10 consecutive days, continuous glucose measurements were compared between the two surgical groups. Significantly lower glucose concentrations were captured from postoperative day 3 in LRYGB versus LSG suggesting that the dose of anti-diabetic medication may need reduction in the former group. Although the intraoperative period was included, there was no data analysis specific to this period. 


\section{Renal}

One study described the impact of obesity on perioperative renal function. Using a matched case-control study technique within the Obesity and Surgical Outcomes database in the US, obese patients undergoing surgery were found to have a $65 \%$ increase in the odds of acute kidney injury (AKI) within 30 days [40]. Postulated theories accounting for this include the difficulties in clinically gauging volume status and accurately measuring blood pressure in obese patients. Both of these topics have been discussed in the cardiovascular section of this review.

\section{Drug Dosing}

Obesity is associated with physiological and anthropometric changes that alter the pharmacokinetics of most drugs. As our focus was on intraoperative monitoring, we included two studies (Table 9) [38, 39] which looked at pointof-care testing and heparin dosing in obesity. In the context of extracorporeal circulation, pointof-care testing (activated clotting time) did not reliably detect the excessively high plasma heparin concentrations often achieved in the obese patient dosed according to total body weight.

\section{Other Considerations}

Temperature management is an important aspect of intraoperative monitoring. Of particular relevance to the obese patient undergoing laparoscopic bariatric surgery is the risk of inadvertent stapling or suturing of the temperature probe, nasogastric tube, or bougie during gastrectomy or gastrojejunostomy. This matter was covered in a questionnaire issued to all bariatric surgeons in Israel, which reported on 17 cases in which one of the above was inadvertently stapled [67].

\section{CONCLUSIONS}

Our narrative review identified 35 studies which pertained to the intraoperative monitoring of obese patients. The majority of these focused on the cardiovascular, neurological, and respiratory systems.

There are a number of limitations to our review. The literature retrieved focused almost exclusively on patients undergoing bariatric surgery, an elective procedure for which patients have been carefully selected and optimised. The in-hospital mortality in the UK for bariatric surgery has been reported at $0.04 \%$, with a complication rate of $2.4 \%$ [68]. A preference for non-invasive monitoring techniques has been described in enhanced recovery protocols for these surgeries [42]. However, the obese patient presenting for non-bariatric, particularly emergency surgery obviously presents a different challenge for which invasive monitoring might be appropriate.

Aside from the diverse surgical settings, it is important to recognise the clinical diversity of the obesity phenotype. The "obesity paradox" has been coined to describe the apparent protective effect of obesity in certain contexts [43]. There is clearly no uniform risk profile within a BMI category.

We applied date restrictions to our search, as both practice and publication (between 2008-2012 and 2013-2018 there was a 94\% increase in PubMed citations on bariatric surgery [7]) have expanded rapidly in this area. Relevant studies might have been missed on this premise.

In summary, there is a tension between the drive towards day surgery and enhanced recovery protocols which both encourage minimally invasive monitoring and the difficulty of accurately applying novel monitoring techniques to this population. This review sought to answer an ostensibly simple question (namely "what intraoperative monitoring techniques have been used in the obese patient?") but ultimately acknowledges the polymorphic nature of both the intraoperative setting and the obesity phenotype itself. 


\section{ACKNOWLEDGEMENTS}

Funding. Open access funding provided by Università degli Studi della Campania Luigi Vanvitelli within the CRUI-CARE Agreement.

Authorship. All named authors meet the International Committee of Medical Journal Editors (ICMJE) criteria for authorship for this article, take responsibility for the integrity of the work as a whole, and have given their approval for this version to be published.

Authors' Contributions. Concept of article: Maria Caterina Pace and Pasquale Sansone. Search strategy: Andrea Haren, Shrijit Nair, Maria Caterina Pace and Pasquale Sansone. Literature search and tabulation: Andrea Haren and Shrijit Nair. Draft of manuscript: Andrea Haren. Revisions: Andrea Haren, Shrijit Nair, Maria Caterina Pace and Pasquale Sansone. Final version reviewed and approved by all authors.

Disclosures. Andrea Haren, Shrijit Nair, Maria Caterina Pace and Pasquale Sansone declare that they have no conflict of interest.

Compliance with Ethics Guidelines. This article is based on previously conducted studies and does not contain any new studies with human participants or animals performed by any of the authors.

Data Availability. All data generated or analyzed during this study are included in this published article.

Open Access. This article is licensed under a Creative Commons Attribution-NonCommercial 4.0 International License, which permits any non-commercial use, sharing, adaptation, distribution and reproduction in any medium or format, as long as you give appropriate credit to the original author(s) and the source, provide a link to the Creative Commons licence, and indicate if changes were made. The images or other third party material in this article are included in the article's Creative Commons licence, unless indicated otherwise in a credit line to the material. If material is not included in the article's Creative Commons licence and your intended use is not permitted by statutory regulation or exceeds the permitted use, you will need to obtain permission directly from the copyright holder. To view a copy of this licence, visit http://creativecommons.org/licenses/by$\mathrm{nc} / 4.0 /$.

\section{REFERENCES}

1. World Health Organization. 2021. Obesity and overweight 2020 [Available from: https://www. who.int/news-room/fact-sheets/detail/obesity-andoverweight.

2. Tsai A, Schumann R. Morbid obesity and perioperative complications. Curr Opin Anaesthesiol. 2016;29(1):103-8.

3. O'Brien PE, Hindle A, Brennan L, Skinner S, Burton $\mathrm{P}$, Smith A, et al. Long-term outcomes after bariatric surgery: a systematic review and meta-analysis of weight loss at 10 or more years for all bariatric procedures and a single-centre review of 20-year outcomes after adjustable gastric banding. Obes Surg. 2019;29(1):3-14.

4. Association of Anaesthetists of Great Britain and Ireland. Peri-operative management of the obese surgical patient 2015. Anaesthesia. 2015;70:859-76.

5. Thorell A, MacCormick AD, Awad S, Reynolds N, Roulin D, Demartines N, et al. Guidelines for perioperative care in bariatric surgery: enhanced recovery after surgery (ERAS) society recommendations. World J Surg. 2016;40(9):2065-83.

6. Petrini F, Di Giacinto I, Cataldo R, Esposito C, Pavoni V, Donato $\mathrm{P}$, et al. Perioperative and periprocedural airway management and respiratory safety for the obese patient: 2016 SIAARTI Consensus. Minerva Anestesiol. 2016;82(12):1314-35.

7. Mechanick JI, Apovian C, Brethauer S, Timothy Garvey W, Joffe AM, Kim J, et al. Clinical practice guidelines for the perioperative nutrition, metabolic, and nonsurgical support of patients undergoing bariatric procedures-2019 update: cosponsored by American association of clinical endocrinologists/American college of endocrinology, the obesity society, American society for metabolic and bariatric surgery, obesity medicine Association, and American society of anesthesiologists. Obesity. 2020;28(4):O1-58. 
8. Association of Anaesthetists of Great Britain and Ireland. Recommendations for standards of monitoring during anaesthesia and recovery 2015. Anaesthesia. 2016;71(1):85-93.

9. Schumann R, Meidert AS, Bonney I, Koutentis C, Wesselink W, Kouz $\mathrm{K}$, et al. Intraoperative blood pressure monitoring in obese patients. Anesthesiology. 2020;134(2):179-88.

10. Rogge DE, Nicklas JY, Haas SA, Reuter DA, Saugel B. Continuous noninvasive arterial pressure monitoring using the vascular unloading technique (CNAP System) in obese patients during laparoscopic bariatric operations. Anesth Analg. 2018;126(2): 454-63.

11. Rogge DE, Nicklas JY, Schön G, Grothe O, Haas SA, Reuter DA, et al. Continuous noninvasive arterial pressure monitoring in obese patients during bariatric surgery: an evaluation of the vascular unloading technique (clearsight system). Anesth Analg. 2019;128(3):477-83.

12. Greiwe G, Tariparast PA, Behem C, Petzoldt M, Herich L, Trepte CJ, et al. Is applanation tonometry a reliable method for monitoring blood pressure in morbidly obese patients undergoing bariatric surgery? Br J Anaesth. 2016;116(6):790-6.

13. Anast $\mathrm{N}$, Olejniczak $\mathrm{M}$, Ingrande J, Brock-Utne J. The impact of blood pressure cuff location on the accuracy of noninvasive blood pressure measurements in obese patients: an observational study. Can J Anaesth = Journal canadien d'anesthesie. 2016;63(3):298-306.

14. Schraverus P, Kuijpers MM, Coumou J, Boly CA, Boer C, van Kralingen S. Level of agreement between cardiac output measurements using Nexfin(®) and thermodilution in morbidly obese patients undergoing laparoscopic surgery. Anaesthesia. 2016;71(12):1449-55.

15. Boly CA, Schraverus P, van Raalten F, Coumou JW, Boer C, van Kralingen S. Pulse-contour derived cardiac output measurements in morbid obesity: influence of actual, ideal and adjusted bodyweight. J Clin Monit Comput. 2018;32(3):423-8.

16. Pösö T, Winsö O, Aroch R, Kesek D. Perioperative fluid guidance with transthoracic echocardiography and pulse-contour device in morbidly obese patients. Obes Surg. 2014;24(12):2117-25.

17. Jain AK, Dutta A. Stroke volume variation as a guide to fluid administration in morbidly obese patients undergoing laparoscopic bariatric surgery. Obes Surg. 2010;20(6):709-15.

18. Ali A, Dorman Y, Abdullah T, Yasa C, Orhan-Sungur $\mathrm{M}$, Akinci IO, et al. Ability of mini-fluid challenge to predict fluid responsiveness in obese patients undergoing surgery in the prone position. Minerva Anestesiol. 2019;85(9):981-8.

19. DeBarros M, Causey MW, Chesley P, Martin M. Reliability of continuous non-invasive assessment of hemoglobin and fluid responsiveness: impact of obesity and abdominal insufflation pressures. Obes Surg. 2015;25(7):1142-8.

20. Holzer A, Sitter B, Kimberger O, Wenzl R, Fleischmann E, Marhofer D, et al. Body mass index does not affect intraoperative goal-directed fluid requirements. Minerva Anestesiol. 2019;85(10): 1071-9.

21. Demirel İ, Bolat E, Altun AY, Özdemir M, Beştaş A. Efficacy of goal-directed fluid therapy via pleth variability index during laparoscopic roux-en-y gastric bypass surgery in morbidly obese patients. Obes Surg. 2018;28(2):358-63.

22. Lorenzen U, Pohlmann $M$, Hansen J, Klose $P$, Gruenewald M, Renner J, et al. Perioperative noninvasive versus semi-invasive cardiac index monitoring in patients with bariatric surgery-a prospective observational study. BMC Anesthesiol. 2020;20(1):196.

23. Soto RG, Davis M, Faulkner MJ. A comparison of the incidence of hypercapnea in non-obese and morbidly obese peri-operative patients using the SenTec transcutaneous pCO(2) monitor. J Clin Monit Comput. 2014;28(3):293-8.

24. Liu S, Sun J, Chen X, Yu Y, Liu X, Liu C. The application of transcutaneous $\mathrm{CO} 2$ pressure monitoring in the anesthesia of obese patients undergoing laparoscopic bariatric surgery. PLOS ONE. 2014;9(4):e91563.

25. Eichler L, Truskowska K, Dupree A, Busch P, Goetz $\mathrm{AE}$, Zöllner C. Intraoperative ventilation of morbidly obese patients guided by transpulmonary pressure. Obes Surg. 2018;28(1):122-9.

26. Nestler C, Simon P, Petroff D, Hammermüller S, Kamrath D, Wolf $S$, et al. Individualized positive end-expiratory pressure in obese patients during general anaesthesia: a randomized controlled clinical trial using electrical impedance tomography. Br J Anaesth. 2017;119(6):1194-205.

27. Tusman G, Groisman I, Fiolo FE, Scandurra A, Arca JM, Krumrick G, et al. Noninvasive monitoring of lung recruitment maneuvers in morbidly obese patients: the role of pulse oximetry and volumetric capnography. Anesth Analg. 2014;118(1):137-44.

28. Tomescu DR, Popescu M, Dima SO, Bacalbasa N, Bubenek-Turconi S. Obesity is associated with decreased lung compliance and hypercapnia during 
robotic assisted surgery. J Clin Monit Comput. 2017;31(1):85-92.

29. Schumann R, Kwater AP, Bonney I, Ladd D, Kim J, Gupta A, et al. Respiratory volume monitoring in an obese surgical population and the prediction of postoperative respiratory depression by the STOPbang OSA risk score. J Clin Anesth. 2016;34: 295-301.

30. Freo U, Carron M, Innocente F, Foletto M, Nitti D, Ori C. Effects of A-line Autoregression Index (AAI) monitoring on recovery after sevoflurane anesthesia for bariatric surgery. Obes Surg. 2011;21(7): 850-7.

31. Demirel I, Yildiz Altun A, Bolat E, Kilinc M, Deniz A, Aksu A, et al. Effect of patient state index monitoring on the recovery characteristics in morbidly obese patients: comparison of inhalation anesthesia and total intravenous anesthesia. J Perianesthesia Nurs Off J Am Soc PeriAnesthesia Nurses. 2020;36(1):69-74.

32. Ibrahim TH, Yousef GT, Hasan AM, Eldesuky HI. Effect of bispectral index monitoring on desflurane consumption and recovery time in morbidly obese patients undergoing laparoscopic sleeve gastrectomy. Anesth Essays Res. 2013;7(1):89-93.

33. Le Gall L, David A, Carles P, Leuillet S, Chastel B, Fleureau C, et al. Benefits of intraoperative analgesia guided by the Analgesia Nociception Index (ANI) in bariatric surgery: an unmatched case-control study. Anaesth Crit Care Pain Med. 2019;38(1): 35-9.

34. Ružman T, Mraović B, Šimurina T, Gulam D, Ružman N, Miškulin M. Transcranial cerebral oxymetric monitoring reduces brain hypoxia in obese and elderly patients undergoing general anesthesia for laparoscopic cholecystectomy. Surg Laparosc Endosc Percutaneous Tech. 2017;27(4):248-52.

35. Torensma $\mathrm{B}$, Martini $\mathrm{CH}$, Boon $\mathrm{M}$, Olofsen $\mathrm{E}$, In 't Veld B, Liem RS, et al. Deep neuromuscular block improves surgical conditions during bariatric surgery and reduces postoperative pain: a randomized double blind controlled trial. PLoS ONE. 2016;11(12):e0167907.

36. Fuchs-Buder T, Schmartz D, Baumann C, Hilt L, Nomine-Criqui C, Meistelman C, et al. Deep neuromuscular blockade improves surgical conditions during gastric bypass surgery for morbid obesity: a randomised controlled trial. Eur J Anaesthesiol. 2019;36(7):486-93.

37. Baete S, Vercruysse G, Vander Laenen M, De Vooght P, Van Melkebeek J, Dylst D, et al. The effect of deep versus moderate neuromuscular block on surgical conditions and postoperative respiratory function in bariatric laparoscopic surgery: a randomized, double blind clinical trial. Anesth Analg. 2017;124(5):1469-75.

38. Haas E, Fischer F, Levy F, Degirmenci SE, Grunebaum L, Kindo M, et al. Identifying optimal heparin management during cardiopulmonary bypass in obese patients: a prospective observational comparative study. Eur J Anaesthesiol. 2016;33(6): 408-16.

39. Vienne M, Haas E, Wipf T, Grunebaum L, Levy F, Sattler L, et al. Adjusted calculation model of heparin management during cardiopulmonary bypass in obese patients: a randomised controlled trial. Eur J Anaesthesiol. 2018;35(8):613-20.

40. Kelz RR, Reinke CE, Zubizarreta JR, Wang M, Saynisch $P$, Even-Shoshan $O$, et al. Acute kidney injury, renal function, and the elderly obese surgical patient: a matched case-control study. Ann Surg. 2013;258(2):359-63.

41. Wysocki M, Szopa M, Stefura T, Dudek A, Torbicz G, Gajewska N, et al. Continuous glucose monitoring in bariatric patients undergoing laparoscopic sleeve gastrectomy and laparoscopic Roux-En-Y gastric bypass. Obes Surg. 2019;29(4):1317-26.

42. Trotta M, Ferrari C, D'Alessandro G, Sarra G, Piscitelli G, Marinari GM. Enhanced recovery after bariatric surgery (ERABS) in a high-volume bariatric center. Surg Obes Relat Dis Off J Am Soc Bariatr Surg. 2019;15(10):1785-92.

43. Kim SH, Després JP, Koh KK. Obesity and cardiovascular disease: friend or foe? Eur Heart J. 2016;37(48):3560-8.

44. Ortiz VE, Kwo J. Obesity: physiologic changes and implications for preoperative management. BMC Anesthesiol. 2015;15:97.

45. Doyle SL, Lysaght J, Reynolds JV. Obesity and postoperative complications in patients undergoing non-bariatric surgery. Obes Rev Off J Int Assoc Study Obes. 2010;11(12):875-86.

46. Kruger GH, Shanks A, Kheterpal S, Tremper T, Chiang CJ, Freundlich RE, et al. Influence of noninvasive blood pressure measurement intervals on the occurrence of intra-operative hypotension. J Clin Monit Comput. 2018;32(4):699-705.

47. Wesselink EM, Kappen TH, Torn HM, Slooter AJC, van Klei WA. Intraoperative hypotension and the risk of postoperative adverse outcomes: a systematic review. Br J Anaesth. 2018;121(4):706-21.

48. Eley VA, Christensen R, Guy L, Dodd B. Perioperative blood pressure monitoring in patients with obesity. Anesth Analg. 2019;128(3):484-91. 
49. Saugel B, Kouz K, Scheeren TWL, Greiwe G, Hoppe $\mathrm{P}$, Romagnoli $\mathrm{S}$, et al. Cardiac output estimation using pulse wave analysis-physiology, algorithms, and technologies: a narrative review. Br J Anaesth. 2021;126(1):67-76.

50. Grocott MP, Dushianthan A, Hamilton MA, Mythen MG, Harrison D, Rowan K. Perioperative increase in global blood flow to explicit defined goals and outcomes after surgery: a cochrane systematic review. Br J Anaesth. 2013;111(4):535-48.

51. Gustafsson UO, Hausel J, Thorell A, Ljungqvist O, Soop M, Nygren J. Adherence to the enhanced recovery after surgery protocol and outcomes after colorectal cancer surgery. Arch Surg (Chicago, Ill: 1960). 2011;146(5):571-7.

52. Myles PS, Bellomo R, Corcoran T, Forbes A, Peyton $P$, Story $D$, et al. Restrictive versus liberal fluid therapy for major abdominal surgery. N Engl J Med. 2018;378(24):2263-74.

53. Chakravartty S, Sarma DR, Patel AG. Rhabdomyolysis in bariatric surgery: a systematic review. Obes Surg. 2013;23(8):1333-40.

54. Sood A, Abdollah F, Sammon JD, Majumder K, Schmid M, Peabody JO, et al. The effect of body mass index on perioperative outcomes after major surgery: results from the national surgical quality improvement program (ACS-NSQIP) 2005-2011. World J Surg. 2015;39(10):2376-85.

55. Neto AS, Hemmes SNT, Barbas CSV, Beiderlinden M, Fernandez-Bustamante A, Futier E, et al. Incidence of mortality and morbidity related to postoperative lung injury in patients who have undergone abdominal or thoracic surgery: a systematic review and meta-analysis. Lancet Respir Med. 2014;2(12):1007-15.

56. Ball L, Hemmes SNT, Serpa Neto A, Bluth T, Canet J, Hiesmayr $\mathrm{M}$, et al. Intraoperative ventilation settings and their associations with postoperative pulmonary complications in obese patients. Br J Anaesth. 2018;121(4):899-908.

57. Bluth T, Serpa Neto A, Schultz MJ, Pelosi P, Gama de Abreu M, Bluth T, et al. Effect of intraoperative high positive end-expiratory pressure (PEEP) with recruitment maneuvers vs low peep on postoperative pulmonary complications in obese patients: a randomized clinical trial. JAMA. 2019;321(23): 2292-305.
58. Griffin J, Terry BE, Burton RK, Ray TL, Keller BP, Landrum AL, et al. Comparison of end-tidal and transcutaneous measures of carbon dioxide during general anaesthesia in severely obese adults. Br J Anaesth. 2003;91(4):498-501.

59. Pandit JJ, Andrade J, Bogod DG, Hitchman JM, Jonker WR, Lucas N, et al. 5th National Audit Project (NAP5) on accidental awareness during general anaesthesia: summary of main findings and risk factors. BJA Br J Anaesth. 2014;113(4):549-59.

60. Membership of the Difficult Airway Society Extubation Guidelines Group MP, Mitchell V, Dravid R, Patel A, Swampillai C, Higgs A. Difficult Airway Society Guidelines for the management of tracheal extubation. Anaesthesia. 2012;67(3):318-40.

61. Plaud B, Baillard C, Bourgain J-L, Bouroche G, Desplanque L, Devys J-M, et al. Guidelines on muscle relaxants and reversal in anaesthesia. Anaesth Crit Care Pain Med. 2020;39(1):125-42.

62. Palsen S, Wu A, Beutler SS, Gimlich R, Yang HK, Urman RD. Investigation of intraoperative dosing patterns of neuromuscular blocking agents. J Clin Monit Comput. 2019;33(3):455-62.

63. Fuchs-Buder T. Neuromuscular monitoring and reversal: responses to the POPULAR study. Lancet Respir Med. 2019;7(2):e3.

64. Coeckelenbergh S, Estebe JP. Antinocicepção intravenosa sem opioides orientada pelo Indice de Analgesia/Nocicepção: relato de caso. Braz J Anesthesiol. 2020;70(6):678-81.

65. Mokdad AH, Ford ES, Bowman BA, Dietz WH, Vinicor F, Bales VS, et al. Prevalence of obesity, diabetes, and obesity-related health risk factors, 2001. JAMA. 2003;289(1):76-9.

66. De Jong A, Rolle A, Souche F-R, Yengui O, Verzilli D, Chanques $\mathrm{G}$, et al. How can I manage anaesthesia in obese patients? Anaesth Crit Care Pain Med. 2020;39(2):229-38.

67. Abu-Gazala S, Donchin Y, Keidar A. Nasogastric tube, temperature probe, and bougie stapling during bariatric surgery: a multicenter survey. Surg Obes Relat Dis Off J Am Soc Bariatr Surg. 2012;8(5): 595-600.

68. The National Bariatric Surgery Registry Committee. 2020. Third Registry Report 2020. 